ARTICLE

https://doi.org/10.1038/s41467-019-12025-8

\title{
Polyunsaturated fatty acid production by Yarrowia lipolytica employing designed myxobacterial PUFA synthases
}

\author{
Katja Gemperlein ${ }^{1}$, Demian Dietrich (10 ${ }^{2}$, Michael Kohlstedt², Gregor Zipf ${ }^{3}$, Hubert S. Bernauer ${ }^{3}$, \\ Christoph Wittmann², Silke C. Wenzel ${ }^{4}$ \& Rolf Müller (1) 1,4
}

Long-chain polyunsaturated fatty acids (LC-PUFAs), particularly the omega-3 LC-PUFAs eicosapentaenoic acid (EPA), docosapentaenoic acid (DPA), and docosahexaenoic acid (DHA), have been associated with beneficial health effects. Consequently, sustainable sources have to be developed to meet the increasing demand for these PUFAs. Here, we demonstrate the design and construction of artificial PUFA biosynthetic gene clusters (BGCs) encoding polyketide synthase-like PUFA synthases from myxobacteria adapted for the oleaginous yeast Yarrowia lipolytica. Genomic integration and heterologous expression of unmodified or hybrid PUFA BGCs yielded different yeast strains with specific LC-PUFA production profiles at promising yield and thus valuable for the biotechnological production of distinct PUFAs. Nutrient screening revealed a strong enhancement of PUFA production, when cells were phosphate limited. This represents, to the best of our knowledge, highest concentration of DHA (16.8\%) in total fatty acids among all published PUFA-producing $Y$. lipolytica strains.

\footnotetext{
${ }^{1}$ Department of Microbial Natural Products, Helmholtz Institute for Pharmaceutical Research Saarland and Helmholtz Centre for Infection Research, Saarbrücken, Germany. ${ }^{2}$ Institute for Systems Biotechnology, Saarland University, Saarbrücken, Germany. ${ }^{3}$ ATG:biosynthetics GmbH, Merzhausen, Germany. ${ }^{4}$ Department of Pharmacy, Saarland University, Saarbrücken, Germany. Correspondence and requests for materials should be addressed to R.M. (email: Rolf.Mueller@helmholtz-hips.de)
} 
A pplication of the hemiascomycetous yeast Yarrowia lipolytica has awakened a strong industrial interest due to its capacity to grow efficiently on hydrophobic substrates (e.g., alkanes, fatty acids, and oils) as a sole carbon source and to produce high amounts of organic acids, especially citric acid ${ }^{1}$. Developments of genetic and cellular tools have contributed to the establishment of $Y$. lipolytica as an amenable host for heterologous protein production ${ }^{2-4}$. Due to its non-pathogenic nature, several processes based on Y. lipolytica were categorized as generally recognized as safe (GRAS) ${ }^{5}$. Yarrowia lipolytica is classified among the oleaginous yeasts because of its ability to accumulate large amounts of lipids (up to $50 \%$ of its cell dry weight $(\mathrm{CDW}))^{6}$. The crucial difference between oleaginous and non-oleaginous yeasts becomes evident during cultivation under nitrogen-limiting conditions ${ }^{7,8}$ : the carbon flux in non-oleaginous yeasts is directed into synthesis of various polysaccharides, whereas in oleaginous yeasts, it is preferentially channeled towards lipid biosynthesis, leading to an accumulation of triacylglycerols within discrete intracellular lipid bodies.

Obviously, Y. lipolytica is a promising host strain for recombinant production of long-chain polyunsaturated fatty acids (LCPUFAs), such as eicosapentaenoic acid (EPA, 20:5, n-3) and docosahexaenoic acid (DHA, 22:6, n-3), which have beneficial effects on health by lowering triglyceride concentrations and blood pressure ${ }^{9}$. As already demonstrated in a previous study, expression of a bifunctional $\Delta^{12} / \omega 3$ desaturase from Fusarium moniliforme in Y. lipolytica yielded $\alpha$-linolenic acid (18:3, $n$-3), a precursor of EPA and DHA, at a concentration of $28.1 \%$ of total fatty acids (TFAs) ${ }^{10}$. Production of $20.2 \% \gamma$-linolenic acid (GLA, $18: 3, n-6)$ of TFAs was achieved by overexpressing $\Delta^{6}$ and $\Delta^{12}$ desaturases from Mortierella alpina ${ }^{11}$. A Y. lipolytica strain that produces EPA at $56.6 \%$ of TFAs was generated by DuPont (USA) ${ }^{12}$. These production levels were reached by overexpression of 30 copies of nine different, codon-optimized genes (20 desaturase genes, eight elongase genes, and two cholinephosphotransferase genes) combined with disruption of four genes, including a gene encoding a peroxisomal biogenesis factor and two genes involved in the lipid metabolism, in the yeast genome. Similarly, DuPont (USA) engineered a Y. lipolytica strain capable of producing $18.3 \% n-3$ docosapentaenoic acid (DPA, 22:5) of TFAs starting from EPA with an introduced $\mathrm{C}_{20 / 22}$ elongase gene and $5.6 \%$ DHA of TFAs proceeding from $n-3$ DPA with an introduced $\Delta^{4}$ desaturase gene ${ }^{13}$.

All these examples are based on the biotransformation of endogenously supplied fatty acids using enzymes from the aerobic PUFA biosynthetic pathways, which employ saturated fatty acids synthesized by the native fatty acid synthase as substrates. In contrast, iteratively acting, multifunctional polyketide synthase (PKS)-like PUFA synthases as found in myxobacteria enable de novo LC-PUFA biosynthesis from acyl-CoA precursors in a multistep process ${ }^{14}$ (Fig. 1a). These multienzyme complexes are encoded by PUFA ( $p f a$ ) biosynthetic gene clusters (BGCs) ${ }^{15}$. The main PUFA produced by the myxobacterium Aetherobacter fasciculatus (SBSr002) represents $\mathrm{DHA}^{16}$, whereas arachidonic acid (AA, 20:4, $n-6$ ) is the predominant PUFA in the myxobacterium Minicystis rosea (SBNa008) ${ }^{17}$ (Fig. 1b). In this study, synthetic biology techniques were applied to establish heterologous expression systems for myxobacterial PUFA synthases in the evolutionary unrelated host $Y$. lipolytica. Artificial pfa BGCs encoding a PUFA synthase plus a $4^{\prime}$-phosphopantetheinyl transferase (PPTase) from A. fasciculatus (SBSr002) ${ }^{14}$ or M. rosea (SBNa008) were redesigned and holistically optimized for $Y$.

a

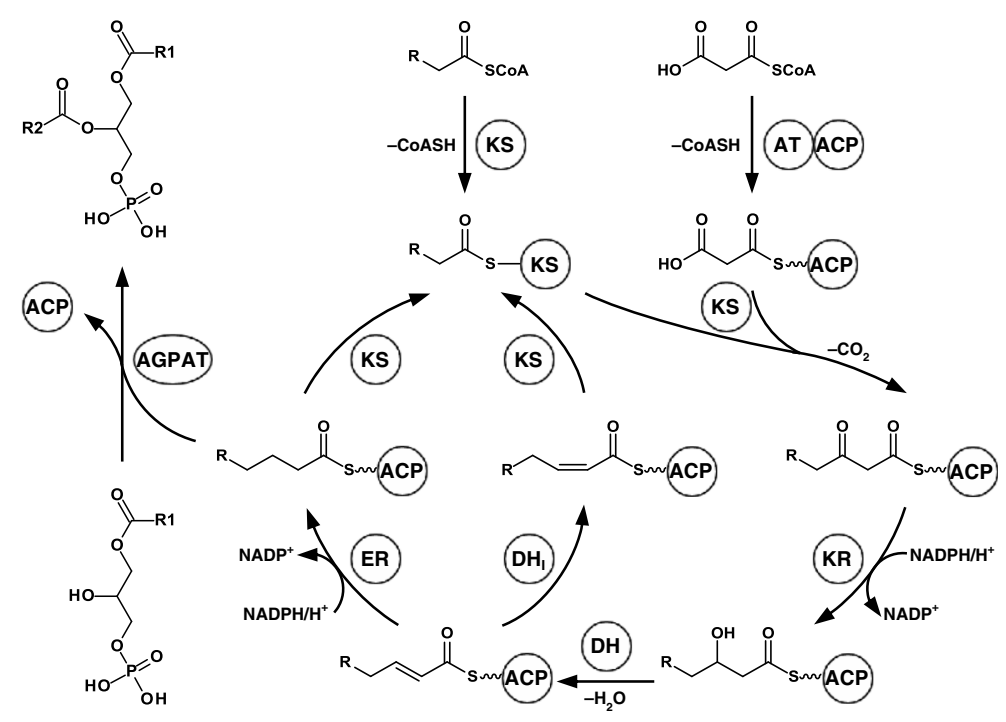

b
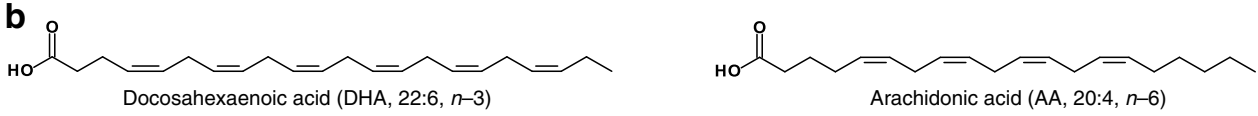

Fig. 1 De novo polyunsaturated fatty acid (PUFA) biosynthesis in myxobacteria by iteratively acting, multifunctional PUFA synthases. a The starter unit acetyl-CoA $(R=H)$ is consecutively elongated with the extender unit malonyl-CoA by several rounds of decarboxylative Claisen condensations, resulting in the extension of the fatty acyl chain by two carbons per cycle. After each round of elongation, the $\beta$-keto group is either fully reduced by ketoreduction, dehydration plus enoylreduction, or only reduced by ketoreduction and dehydration, giving rise to the trans double bond, which is then isomerized to synthesize an acyl chain bearing methylene-interrupted cis double bonds. After reaching its final length, the fatty acyl chain is presumably used for acylation of the 2-position of 1-acylglycerol-3-phosphate. KS, ketosynthase of Pfa2 and Pfa3; AT, acyltransferase of Pfa2 and Pfa3; ACP, acyl carrier protein of Pfa2; KR, ketoreductase of Pfa2; $\mathrm{DH}$, dehydratase of Pfa2 and Pfa3; $\mathrm{DH}_{1}$, dehydratase/isomerase of Pfa3; ER, enoylreductase of Pfa1; AGPAT, 1acylglycerol-3-phosphate O-acyltransferase of Pfa3. b Structures of the main PUFAs produced by the myxobacteria Aetherobacter fasciculatus (SBSrO02) and Minicystis rosea (SBNa008) 
lipolytica, including codon bias adaptation, followed by synthesis and assembly of the respective DNA building blocks. Moreover, diverse hybrid $p f a$ BGCs were constructed from these two pathways. Chromosomal integration of the synthetic BGCs yielded transgenic $Y$. lipolytica strains that specifically produce LCPUFAs, such as AA, EPA, DPA, and DHA.

\section{Results}

Design of a synthetic BGC encoding a DHA-type PUFA synthase. The establishment of heterologous expression platforms for recombinant LC-PUFA production using the DPA/DHA-type $p f a$ BGC from A. fasciculatus (SBSr002) in earlier studies ${ }^{14,18}$ served as proof of principle and paved the way for the development of a phylogenetically more distant host organism, exhibiting several advantageous attributes. In this context, the oleaginous yeast $Y$. lipolytica was focused on as expression host of choice, since, in contrast to the myxobacterial LC-PUFA producers, it features GRAS designation, fast growth characteristics, accessibility for genetic manipulation, and ability to accumulate large amounts of lipids. For the heterologous expression of the $p f a$ BGC encoding the DPA/DHA-type PUFA synthase plus a PPTase from A. fasciculatus $(\mathrm{SBSr} 002)^{14,16}$ in the oleaginous yeast $Y$. lipolytica, synthetic versions of the three $p f a$ genes and $p p t$ were created $(\rightarrow$ BGC version C1_V1; Fig. 2a). Each coding sequence is flanked by the strong hybrid hp4d promoter ${ }^{3}$ and the LIP2 terminator for $Y$. lipolytica to construct single transcription units. Furthermore, non-coding sequences were attached, connecting each transcription unit by $200 \mathrm{bp}$ intergenic linkers. During sequence modulation processes, the myxobacterial genes were subjected to the algorithms of the proprietary evoMAG software by ATG:biosynthetics ${ }^{19}$. The software applies concepts of genetic evolutionary algorithms $\mathrm{s}^{20,21}$ to generate sequences that take predefined multivariate sequence parameter values like codon usage frequencies, RNA secondary structures, and sequence motifs into account. Degeneracy of the genetic code allows for the substitution of synonymous codons by silent mutations to modulate the artificial sequence without altering the native amino acid sequence for formal functional biodesigns. The sequence of BGC $\mathrm{C} 1$ V1 was designed with the intention to improve the expression by altering the translational elongation profile. An artificial codon usage table was generated from Y. lipolytica genome reference data by excluding codons below a predefined synonymous fraction threshold and subsequent normalization of the codon table. Afterwards, each codon position was varied iteratively according to the artificial codon table until the course of the local codon adaptation index was smoothened, and all predetermined scoring requirements were fulfilled (details of the sequence design can be found in Supplementary Note 1, Supplementary Figs. 1, 3, 6, 7, and 9-11, and Supplementary Tables 1, 4-8, 14-19). For practical reasons, the $20.2 \mathrm{~kb}$ BGC was dissected into smaller DNA fragments, which were supplied by gene synthesis companies. Constructive sequence requirements for pathway assembly from the synthetic DNA building blocks and for future interchangeability of inter- and intragenic regions were specified and implemented by insertion of unique restriction enzyme sites at any required position within the sequence (Fig. 2a). In parallel, interfering restriction sites had to be eliminated from the sequences.

Random genomic integration of the artificial $p f a$ BGC. The synthetic pathway C1_V1 encoding PUFA synthases plus a PPTase from A. fasciculatus was reconstituted by assembly of the respective DNA building blocks. For the final expression construct, a plasmid backbone was constructed on the basis of the Y. lipolytica shuttle vector pINA1312 to enable random integration of typically one copy of the transgenes into the genome of $Y$. lipolytica ${ }^{2}$. The yeast a

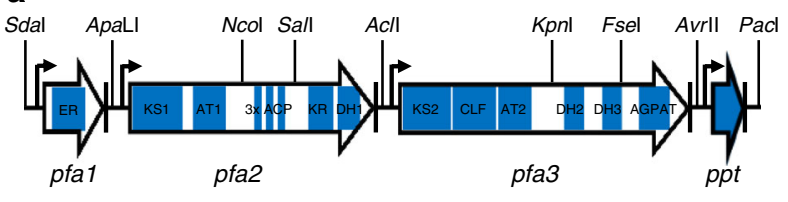

b

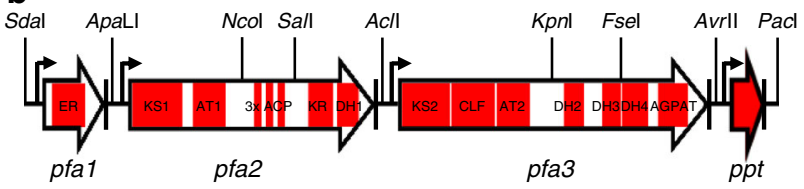

c

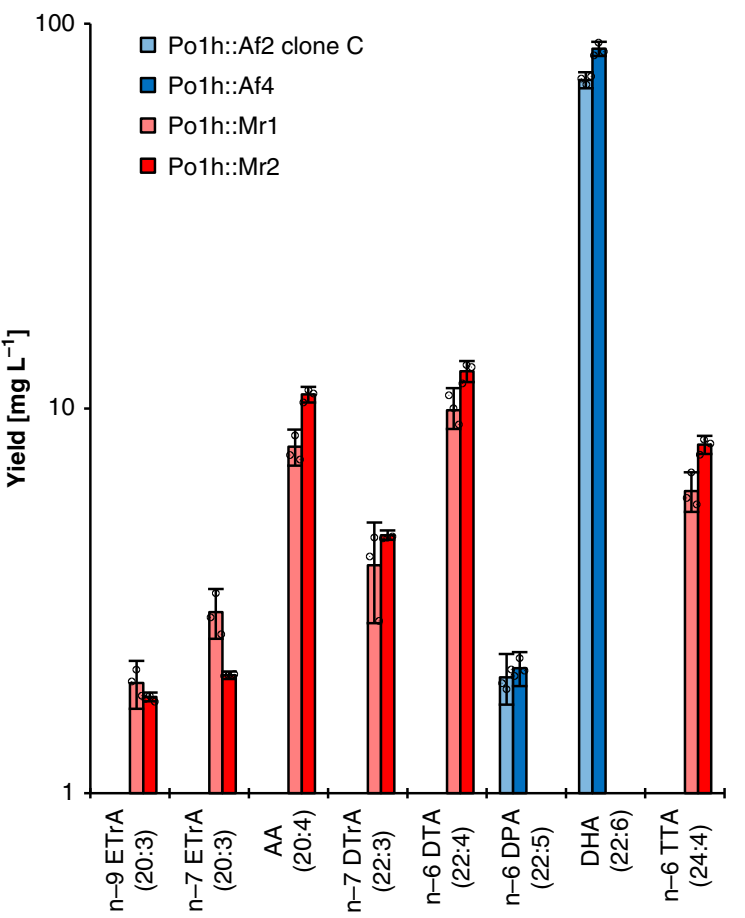

Fig. 2 Artificial pfa biosynthetic gene clusters encoding myxobacterial PUFA synthases for LC-PUFA production in Yarrowia lipolytica Po1h. a Synthetic pfa BGC (20.2 kb) version C1_V1 or C1_V2 comprising genes pfa1, pfa2, and pfa3 encoding the DPA/DHA-type PUFA synthase plus gene ppt encoding the 4'phosphopantetheinyl transferase (PPTase) from Aetherobacter fasciculatus (SBSr002) adapted for the oleaginous yeast Yarrowia lipolytica. b Synthetic pfa BGC (21.1 kb) version C3 or C3_mod 5' comprising genes pfa1, pfa2, and pfa3 encoding the AA/DTA-type PUFA synthase plus gene ppt encoding the PPTase from Minicystis rosea (SBNa008) adapted for Y. lipolytica. c LC-PUFAs produced by $Y$. lipolytica Po1h::Af4 (harboring synthetic pfa BGC version C1_V1), Y. lipolytica Po1h::Af7 (harboring synthetic pfa BGC version C1_V2), Y. lipolytica Polh::Mr1 (harboring synthetic pfa BGC version C3), and Y. lipolytica Polh::Mr2 (harboring synthetic pfa BGC version C3_mod 5'). Each coding sequence of all clusters is flanked by the strong hybrid hp4d promoter ${ }^{3}$ and the LIP2 terminator. Unique restriction enzyme sites are present at specific positions for pathway assembly and for interchangeability of inter- and intragenic regions. ER, enoylreductase; KS1+KS2, ketosynthases; AT1+AT2, acyltransferases; $A C P$, acyl carrier protein; $K R$, ketoreductase; $D H 1+D H 4$, polyketide synthase (PKS)-like dehydratases; $C L F$, chain length factor; $\mathrm{DH} 2$ +DH3, FabA-like dehydratases/isomerases; AGPAT, 1-acylglycerol-3phosphate $\mathrm{O}$-acyltransferase. Cultivations were carried out in $10 \mathrm{~mL}$ YNBG medium $+50 \mathrm{mM}$ potassium phosphate buffer $\mathrm{pH} 6.8$ at $28^{\circ} \mathrm{C}$ and $200 \mathrm{rpm}$ for $168 \mathrm{~h}$. The indicated values are means and s.d. of three biological replicates, presented on a logarithmic scale. Source data are provided as a Source Data file. ETrA, eicosatrienoic acid; $A A$, arachidonic acid; $D \operatorname{Tr} A$, docosatrienoic acid; DTA, docosatetraenoic acid; DPA, docosapentaenoic acid; DHA, docosahexaenoic acid; TTA, tetracosatetraenoic acid 
cassette (DNA sequence devoid of bacterial sequences) of the resulting plasmid pAf2 was liberated via hydrolysis by a restriction endonuclease and transferred into $Y$. lipolytica Polh ${ }^{4}$. In an initial screening, 38 transformants of Y. lipolytica Polh::Af2 were cultivated in triplicates and their fatty acid methyl esters (FAMEs) were isolated by direct transesterification as well as analyzed by gas chromatography-mass spectrometry (GC-MS). Eleven clones were shown to produce DHA, with transgenic strain Y. lipolytica Polh:: Af2 clone $\mathrm{C}$ as the best producer. It was re-cultivated in triplicates in $10 \mathrm{~mL}$ YNBG medium (heterologous LC-PUFA production was superior in minimal YNBG medium compared to rich, complex YPD medium) at $28^{\circ} \mathrm{C}$ for $168 \mathrm{~h}$, and the FAMEs were isolated and analyzed. DHA was produced at a concentration of $71.4 \mathrm{mg} \mathrm{L}^{-1}$ or $9.5 \%$ of TFAs and $9.8 \mathrm{mg} \mathrm{g}^{-1} \mathrm{CDW}$, and also some minor amounts of $n-6$ DPA and $n-3$ DPA were produced (Fig. 2c). Shotgun genome sequencing of $Y$. lipolytica Polh::Af2 clone C revealed the hypothetical gene YALI0_C05907g as integration site for construct pAf2. These results were a proof of concept for the heterologous expression of artificial $p f a$ BGCs in Y. lipolytica and served as basis for further engineering of the LC-PUFA expression platform.

Site-specific genomic integration of the artificial pfa BGC. Gene expression in eukaryotes is a complex process regulated at multiple levels. Transcription of transgenes is not only influenced by recombinant promoters and regulatory DNA elements but also by the spatial positioning of the transgenes within the genome $\mathrm{e}^{22,23}$. In the course of the present study, locus YALI0_C05907g has emerged as an integration site that enables a good expression of recombinant $p f a$ BGCs. Therefore, plasmid pKG2-PIS, allowing for site-specific integration of the $p f a$ BGC into locus YALI0_C05907g via double crossover, was constructed. The backbone of the plasmid pAf2 was exchanged for plasmid pKG2-PIS, and the yeast cassette of the resulting plasmid pAf4 was liberated via hydrolysis by restriction endonucleases and transferred into Y. lipolytica Polh ${ }^{4}$. After genotypic verification, transgenic strain Y. lipolytica Polh::Af4 was cultivated in triplicates as described above, followed by isolation and analysis of the FAMEs. As expected, PUFA production was achieved at a similar level (even slightly higher) as observed for Y. lipolytica Polh::Af2 clone C: DHA was detected at a concentration of $86.1 \mathrm{mg} \mathrm{L}^{-1}$ or $10.5 \%$ of TFAs and $12.8 \mathrm{mg} \mathrm{g}^{-1} \mathrm{CDW}$, and $n-6$ DPA plus $n-3$ DPA in some minor amounts were also detected (Fig. $2 \mathrm{c}$ and Supplementary Table 9).

Besides the sequence design for BGC C1_V1, we aimed at testing the effect of an alternative gene optimization approach, a codon harmonization resembling strategy, on recombinant LCPUFA production in $Y$. lipolytica. Therefore, BGC version C1_V2, encoding PUFA synthases plus a PPTase from $A$. fasciculatus, was designed. The constructional sequence design and the assembly of the building blocks were performed analogous to BGC C1_V1 (details of the sequence design can be found in Supplementary Note 1). Interestingly, $Y$. lipolytica Po1h::Af7, containing BGC C1_V2, produces DHA, n-6 DPA, and $n-3$ DPA in comparable amounts as Y. lipolytica Polh::Af4 (Supplementary Table 9).

Design of a synthetic BGC encoding a DTA-type PUFA synthase. In addition to the artificial $p f a$ BGCs encoding the DPA/ DHA-type PUFA synthase from A. fasciculatus, the $p f a$ BGCs encoding PUFA synthases plus a PPTase originating from $M$. rosea $(\mathrm{SBNa008})^{17}$ was designed for heterologous expression in the oleaginous yeast $Y$. lipolytica. The constructional and functional sequence design of the resulting $21.1 \mathrm{~kb} p f a$ BGC C3 (Fig. 2b) was performed analogous to the design of BGC C1_V1 (details of the sequence design can be found in Supplementary
Note 1, Supplementary Figs. 2, 4, and 8, and Supplementary Tables 1 and 2). Six building blocks containing the three $p f a$ coding sequences as well as the $p p t$ coding sequence flanked by hp4d promoters and LIP2 terminators, plus intergenic linker sequences were synthesized, assembled, and finally cloned into plasmid pKG2-PIS. The yeast cassette of the resulting plasmid pMr1 was transferred into Y. lipolytica Polh and clones with correct integration of the transgenes into the preferred integration site (YALI0_C05907g) were identified. After three independent cultivations of strain Y. lipolytica Polh::Mr1 as described above, the FAMEs were isolated and analyzed. The transgenic strain produces mainly $n-6$ LC-PUFAs, such as AA $\left(8.0 \mathrm{mg} \mathrm{L}^{-1}\right), n-6$ docosatetraenoic acid (DTA, 22:4) $\left(9.9 \mathrm{mg} \mathrm{L}^{-1}\right)$, and $n-6$ tetracosatetraenoic acid (TTA, 24:4) $\left(6.1 \mathrm{mg} \mathrm{L}^{-1}\right)$ (Fig. $2 \mathrm{c}$ and Supplementary Table 9). Calculations of the opening energies within the translation initiation sites of genes $p f a 1, p f a 2, p f a 3$, and $p p t$ of BGC C3 revealed the potential for improvement of the ribosomal access to the translational initiation region on the mRNA level. Consequently, the $5^{\prime}$ coding regions of all four genes were redesigned (all details of the sequence design can be found in the Supplementary Note 1, Supplementary Fig. 5, and Supplementary Tables 2 and 3). DNA fragments carrying the calculated silent mutations in the $5^{\prime}$ coding sequences of the genes were synthesized, and the $5^{\prime}$ coding regions of all the genes of BGC C3 were exchanged for the newly adapted sequences, yielding BGC C3_mod 5'. After transfer of the yeast cassette of the generated plasmid pMr2 into the preferred integration site (YALI0_C05907g) in the genome of Y. lipolytica Polh, strain $Y$. lipolytica Polh::Mr2 was cultivated in triplicates as described above. Subsequently, the FAMEs were isolated and analyzed. Compared to strain Y. lipolytica Polh::Mr1, LC-PUFA production yields could be indeed increased by $22 \%$ using $Y$. lipolytica Polh:: Mr2 (10.9 $\mathrm{mg} \mathrm{L}^{-1} \mathrm{AA}, 12.5 \mathrm{mg} \mathrm{L}^{-1} n-6$ DTA, and $8.1 \mathrm{mg} \mathrm{L}^{-1} n-6$ TTA; Fig. 2c and Supplementary Table 9). The product specificity of the PUFA synthase from $M$. rosea differs widely from that of $A$. fasciculatus with respect to chain length as well as the number and position of double bonds of the produced LC-PUFAs, although the structure of both $p f a$ BGCs is very similar (Fig. 2). The most obvious difference between these two multienzyme complexes is the presence of a PKS-type $\mathrm{DH}$ domain (DH4) in Pfa3 from $M$. rosea, which is absent in the homologous protein from $A$. fasciculatus.

Heterologous expression of hybrid $p f a$ BGCs. The factors determining the nature of the produced LC-PUFAs within the (myxo)bacterial PUFA synthases have not yet been elucidated. The production of hybrid PUFA synthases or of PUFA synthases with functional knockouts of domains can contribute to the dissection of the molecular basis of the specificity of PUFA synthase-catalyzed reactions. Consequently, several versions of chimeric $p f a$ BGCs were constructed by using the artificial DPA/ DHA- and AA/DTA-type $p f a$ BGCs. The yeast cassettes of the resulting plasmids described below were transferred into $Y$. lipolytica Polh and clones with correct integration of the transgenes into the preferred integration site (YALI0_C05907g) were identified. After cultivation of the generated Y. lipolytica strains in triplicates as described above, the FAMEs were isolated and analyzed.

At first, the effect of the exchange of the ER domain encoded by $p f a 1$ on the PUFA production profile was examined. Therefore, plasmid pHyb7, encoding a chimeric PUFA synthase consisting of protein Pfal of the DPA/DHA-type PUFA synthase from A. fasciculatus and proteins Pfa2 and Pfa3 of the AA/DTAtype PUFA synthase from $M$. rosea, was constructed. The LCPUFA species produced by this hybrid PUFA synthase are highly 
similar to those produced by the AA/DTA-type PUFA synthase of $M$. rosea. Obviously, the nature of the produced LC-PUFAs is not influenced by the ER domain encoded by $p f a 1$. Next, the effect of the exchange of the gene $p f a 3$ or of selected catalytic domains encoded by $p f a 3$ was evaluated. The exchange of the entire gene $p f a 3$ from the DPA/DHA-type $p f a$ BGC for gene $p f a 3$ from the AA/DTA-type $p f a$ BGC $(\rightarrow$ plasmid pSynHybPfaPpt1; Fig. 3a) resulted in the predominant production of the LC-PUFAs EPA and $n-3$ DPA, which are (almost) not produced by the two native PUFA synthases (Fig. 3b). The replacement of domains KS2-CLF-AT2-DH2-DH3 from gene pfa3 of the DPA/DHA-type $p f a$ BGC with the homologous domains from gene $p f a 3$ of the AA/ DTA-type $p f a$ BGC plus insertion of domain DH4 from gene $p f a 3$ of the AA/DTA-type pfa BGC led to plasmid pHyb2a (Fig. 3a). Plasmid pHyb5a was constructed by the exchange of domains DH2-DH3 from gene $p f a 3$ of the DPA/DHA-type $p f a$ BGC for the homologous domains from gene $p f a 3$ of the AA/DTA-type $p f a$ BGC plus insertion of domain DH4 from gene $p f a 3$ of the AA/ DTA-type $p f a$ BGC (Fig. 3a). As in the case of pHyb1, Y. lipolytica Polh transformants containing the hybrid $p f a$ BGCs originating either from plasmid $\mathrm{pHyb2a}$ or from plasmid $\mathrm{pHyb} 5 \mathrm{a}$ produce EPA and $n-3$ DPA as major products (Fig. $3 \mathrm{~b}$ ). Remarkably, the FabA-like DH domains DH2 and DH3 and the PKS-like DH domain DH4 seem to have a big impact on the PUFA product specificity of Pfa3. Subsequently, the effect of the exchange of the AGPAT domain of $p f a 3$ and/or the insertion of the DH4 domain was investigated. Both the exchange of the AGPAT domain from gene $p f a 3$ of the DPA/DHA-type $p f a$ BGC for the AGPAT domain from gene $p f a 3$ of the AA/DTA-type $p f a \mathrm{BGC}$ and the insertion of domain DH4 from gene $p f a 3$ of the AA/DTA-type $p f a$ BGC led to plasmid pHyb6 (Fig. 3c). The resulting strain Y. lipolytica Polh:: Hyb6 exhibits production of its main product $n-3$ DPA at a concentration of $48.0 \mathrm{mg} \mathrm{L}^{-1}$ or $5.7 \%$ of TFAs and $7.0 \mathrm{mg} \mathrm{g}^{-1}$ $\mathrm{CDW}$ (Fig. 3d). The insertion of only domain DH4 from gene pfa3 of the AA/DTA-type $p f a$ BGC into the DPA/DHA-type $p f a$ BGC $(\rightarrow$ plasmid pHyb6b; Fig. 3c) altered the product spectrum towards n-3 DPA and DHA as major products (Fig. $3 \mathrm{~d}$ ). However, the inactivation of the DH4 domain in plasmid pHyb6b by converting the active site histidine residue into an alanine residue $(\rightarrow$ plasmid pHyb6b-H2270A; Fig. 3c) results in a production profile, which is again identical to that of the DPA/DHA-type PUFA synthase from A. fasciculatus (Figs. $2 \mathrm{c}$ and $3 \mathrm{~d}$ ). Hence, it can be stated that insertion of domain DH4 has a large impact on the LC-PUFA product specificity of Pfa3.

Apart from the investigations on gene $p f a 3$, the effect of the exchange of either the entire gene $p f a 2$ or, based on the observations made with the $\mathrm{DH}$ domains of $p f a 3$, of the exchange of only domain DH1 was examined. Hence, gene $p f a 2$ or domain DH1 from the DPA/DHA-type $p f a$ BGC was exchanged for the homologous gene or domain from the AA/ DTA-type $p f a$ BGC. Interestingly, in both cases the product spectrum is altered towards $\omega-6$ LC-PUFAs with $n-6$ DPA as the main product (Fig. 3e, f). Thus, the nature and the ratio of the produced LC-PUFAs are identical either when the complete gene has been replaced ( $\rightarrow$ plasmid pHyb8; Fig. 3e) or when only domain $\mathrm{DH} 1$ has been exchanged ( $\rightarrow$ plasmid pHyb9; Fig. 3e). It seems that this PKS-type DH domain is one of the domains affecting the preference of Pfa2 for the production of certain LCPUFA species. Vice versa, the replacement of domain DH1 from gene $p f a 2$ of the AA/DTA-type $p f a$ BGC by the homologous domain from gene $p f a 2$ of the DPA/DHA-type $p f a$ BGC $(\rightarrow$ plasmid pHyb15; Fig. $3 \mathrm{~g}$ ) changes the production profile towards $\omega$-3 LC-PUFAs with $n-3$ DPA as the major product (Fig. $3 \mathrm{~h}$ ).

From all of these data, the conclusion can be drawn that the control of chain length and number/position of double bonds seems to require a very complex interplay of multiple functional domains with the $\mathrm{DH}$ domains as important interaction partners. From a biotechnological point of view, the expression of hybrid $p f a$ BGCs is especially beneficial, as it exhibits the potential for the production of valuable LC-PUFAs, such as EPA and n-3 DPA, which are (almost) not produced by the two native PUFA synthases.

Optimization of DHA production by medium design. In order to improve overall productivity of PUFA in Y. lipolytica, different medium compositions and culture conditions were compared to investigate their impact on key performance criteria of the DHA producer Y. lipolytica Polh::Af4, such as titer, productivity, and selectivity. The basic setup, using glucose together with YNB and a phosphate buffer yielded about $20 \mathrm{mg} \mathrm{L}^{-1}$ DHA, which represented about $6 \%$ of TFAs (Fig. 4). Additional analyses revealed that glycerol was particularly efficient to drive DHA biosynthesis (Fig. 4a). The DHA titer achieved was about twice as high as compared to glucose. Surprisingly, a restriction in the availability of phosphate turned out to be important for production efficiency. On glucose as well as on glycerol, the DHA titer was significantly higher, when the phosphate buffer was replaced by MES (2-(N-morpholino)ethanesulfonic acid). Furthermore, phosphate limitation enhanced biosynthetic selectivity with regard to the total DHA content in the biomass as well as the fraction of DHA among the TFAs (Fig. 4b). A further reduction of phosphate by lower levels of YNB finally enabled the accumulation of more than $100 \mathrm{mg} \mathrm{L}^{-1}$ DHA and a high DHA content of $16.6 \%$ of TFAs (Fig. 4d). In contrast, nitrogen limitation was not necessarily required, different to the common picture. As an example, poor DHA accumulation resulted when glucosegrown cells early depleted ammonium, whereas the production was high in the presence of excess ammonium during the whole cultivation time (Supplementary Fig. 12). Generally, highproducing conditions resulted in the accumulation of citrate, which was partially re-consumed in later stages of the cultivation. Acetate, tested as an alternative carbon source, triggered an even higher selectivity of $16.8 \%$, but yielded lower levels of biomass (Supplementary Table 10).

Fed-batch production process for DHA. To assess performance under industrially relevant conditions, we benchmarked the DHA producer Y. lipolytica Po1h::Af4 in a fed-batch process, which was operated under phosphate-limiting conditions (Fig. 5). Glucose and glycerol were tested in parallel setups as carbon source. On both substrates, the strain grew fast during the initial batch phase and reached a biomass level of about $30 \mathrm{~g} \mathrm{~L}^{-1}$. During this phase, DHA was not accumulated. Glycerol catabolism resulted in a slight accumulation of citrate, which was not observed in the glucose-based process. Both substrates were efficiently consumed during the first $48 \mathrm{~h}$. Likely triggered by the limitation of phosphate, growth then stopped and the cells switched into a producing mode. The DHA level increased to more than $350 \mathrm{mg} \mathrm{L}^{-1}$ on glucose after $300 \mathrm{~h}$. On glycerol, the final titer was slightly lower $\left(300 \mathrm{mg} \mathrm{L}^{-1}\right)$. The glucose-based process was also superior with regard to the fraction of DHA formed. The DHA content gradually increased during the process and reached a final value of $>10 \%$. The imposed feed rate enabled low substrate levels during the entire feed phase, which, however, were still high enough to keep still cells in their producing mode. Citrate accumulated to some extent during the feed phase. Other by-products were not formed.

\section{Discussion}

In this project, we established expression systems for recombinant LC-PUFA production in the oleaginous yeast $Y$. lipolytica based 
a
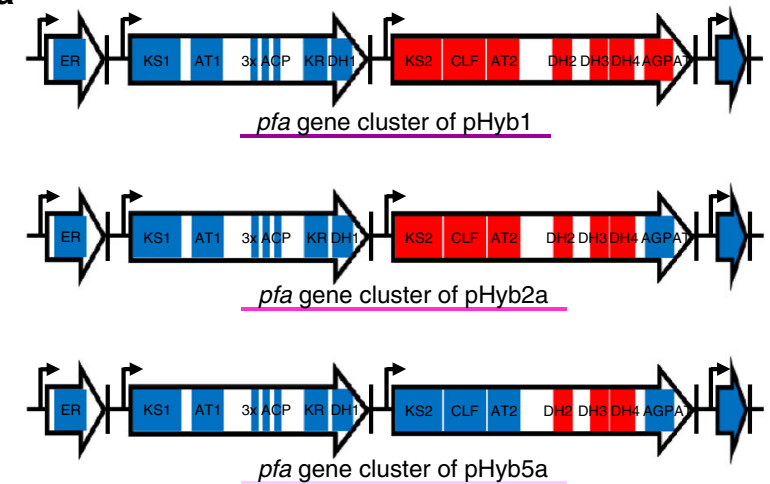

b

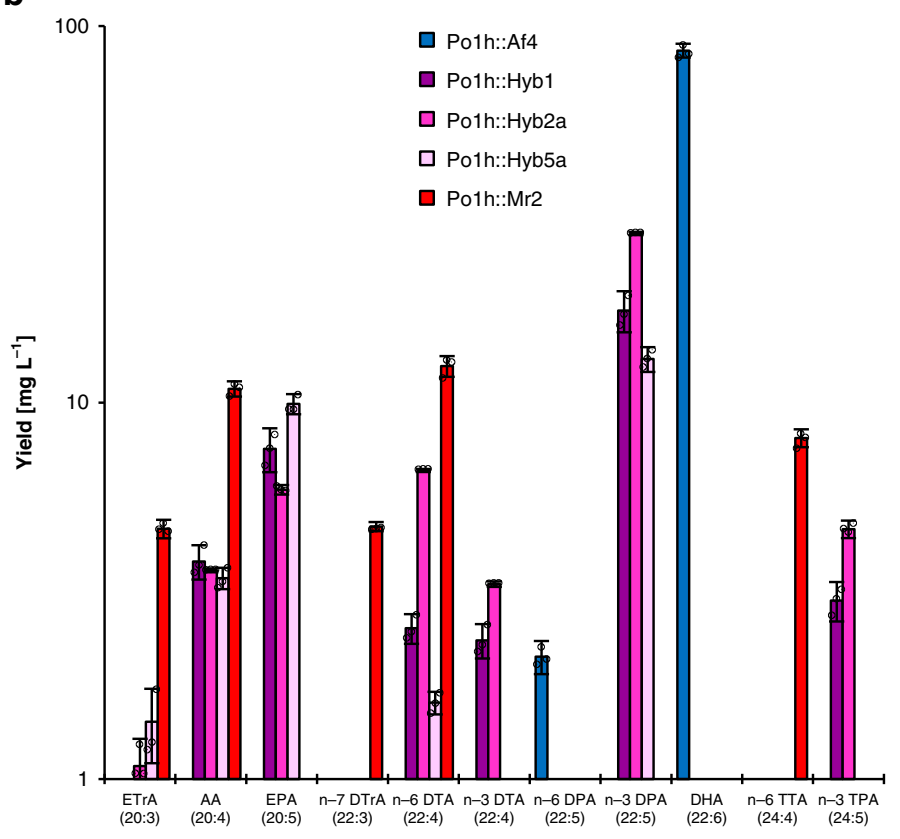

c
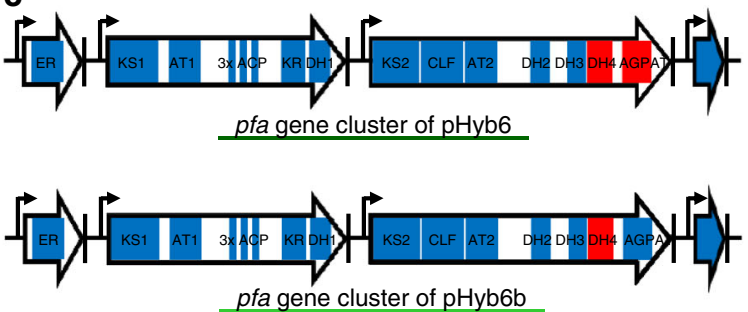

pfa gene cluster of pHyb6b

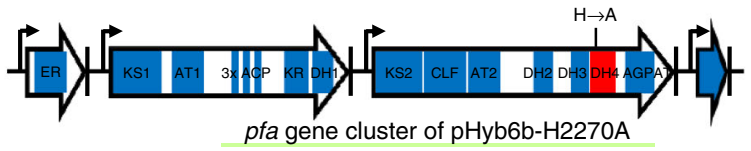

d

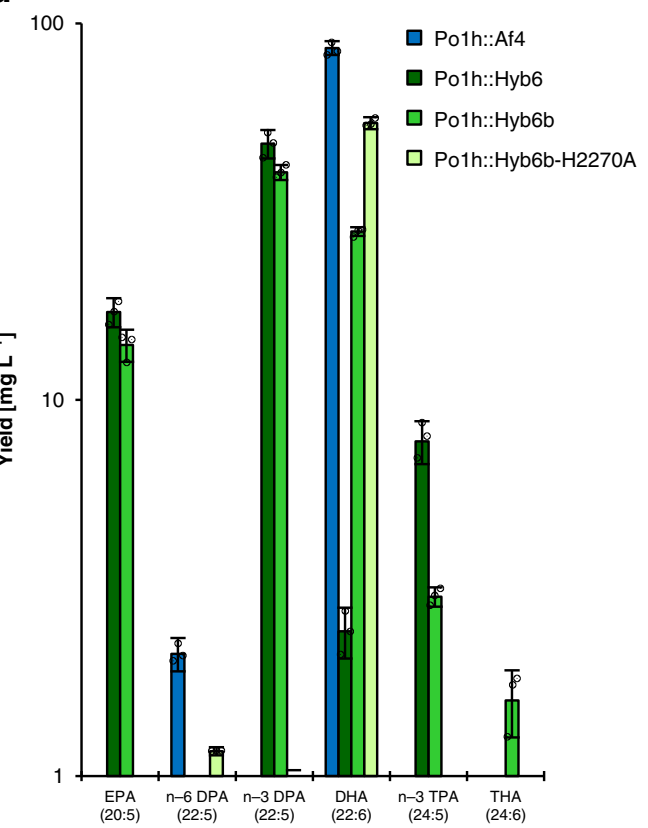

e
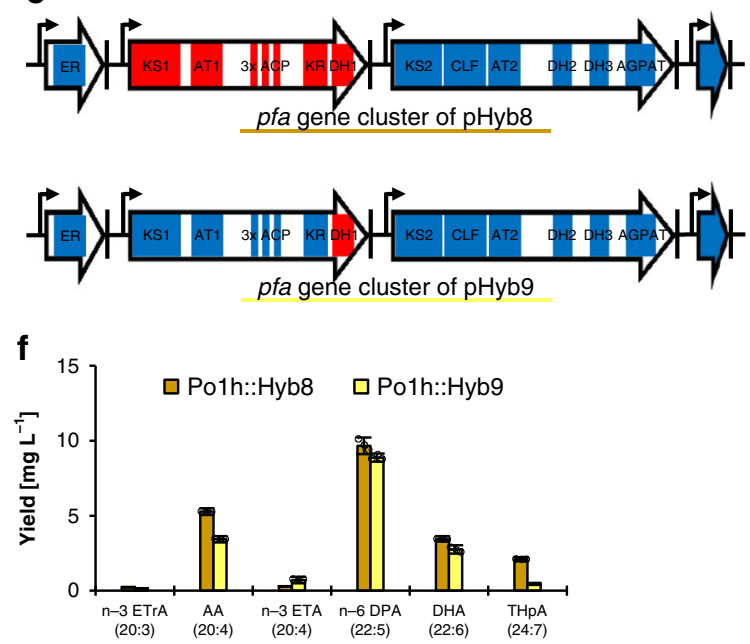

on myxobacterial PUFA biosynthetic machineries, employing methods of Synthetic Biology. By the usage of synthetic, codonoptimized PUFA BGCs encoding homologous PUFA synthases from two myxobacterial species, Y. lipolytica production strains with completely different LC-PUFA production profiles regarding chain length as well as the number and position of the double bonds could be generated. Remarkably, one of these Y. lipolytica
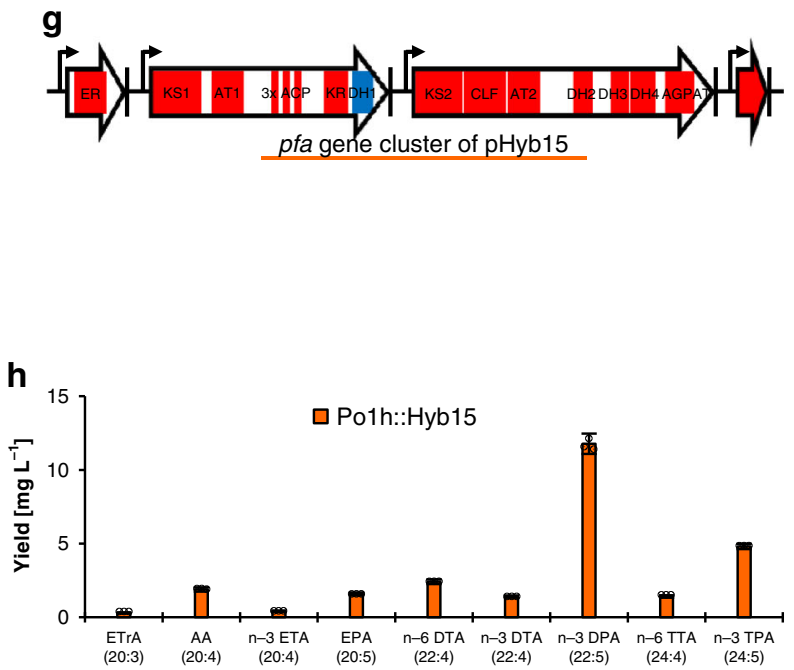

strains is able to produce the valuable long-chain $\omega-3$ fatty acid DHA at a concentration of $350 \mathrm{mg} \mathrm{L}^{-1}$ or $16.8 \%$ of TFAs under improved fermentation conditions. DHA is critical for normal development and functioning of the brain ${ }^{24}$ and is predominantly present in marine fish and the corresponding fish oils ${ }^{25}$, a nonsustainable source. The exchange of single genes or domainsespecially the DH domains-between the two synthetic $p f a$ BGCs 
Fig. 3 LC-PUFAs produced by Yarrowia lipolytica Polh using chimeric PUFA synthases encoded by artificial pfa biosynthetic gene clusters. a Hybrid pfa BGCs located on plasmid pHyb1, plasmid pHyb2a, and plasmid pHyb5a. b LC-PUFAs produced by Y. lipolytica Po1h::Hyb1, Y. lipolytica Po1h::Hyb2a, and Y. lipolytica Po1h::Hyb5a. c Hybrid pfa BGCs located on plasmid pHyb6, plasmid pHyb6b, and plasmid pHyb6b-H2270A. d LC-PUFAs produced by Y. lipolytica Po1h::Hyb6, Y. lipolytica Po1h::Hyb6b, and Y. lipolytica Po1h::Hyb6b-H2270. e Hybrid pfa BGCs located on plasmid pHyb8 and plasmid pHyb9. f LC-PUFAs produced by Y. lipolytica Po1h::Hyb8 and Y. lipolytica Po1h::Hyb9. g Hybrid pfa BGCs located on plasmid pHyb15. h LC-PUFAs produced by Y. lipolytica Po1h:: Hyb15. Domains from the synthetic DPA/DHA-type pfa BGC are shown in blue; domains from the synthetic AA/DTA-type pfa BGC are shown in red. Cultivations were carried out in $10 \mathrm{~mL}$ YNBG medium $+50 \mathrm{mM}$ potassium phosphate buffer $\mathrm{pH} 6.8$ at $28^{\circ} \mathrm{C}$ and $200 \mathrm{rpm}$ for $168 \mathrm{~h}$. The indicated values are means and s.d. of three biological replicates. Source data are provided as a Source Data file. ETrA, eicosatrienoic acid; AA, arachidonic acid; ETA, eicosatetraenoic acid; EPA, eicosapentaenoic acid; DTrA, docosatrienoic acid; DTA, docosatetraenoic acid; DPA, docosapentaenoic acid; DHA, docosahexaenoic acid; TTA, tetracosatetraenoic acid; TPA, tetracosapentaenoic acid; THA, tetracosahexaenoic acid; THpA, putative tetracosaheptaenoic acid
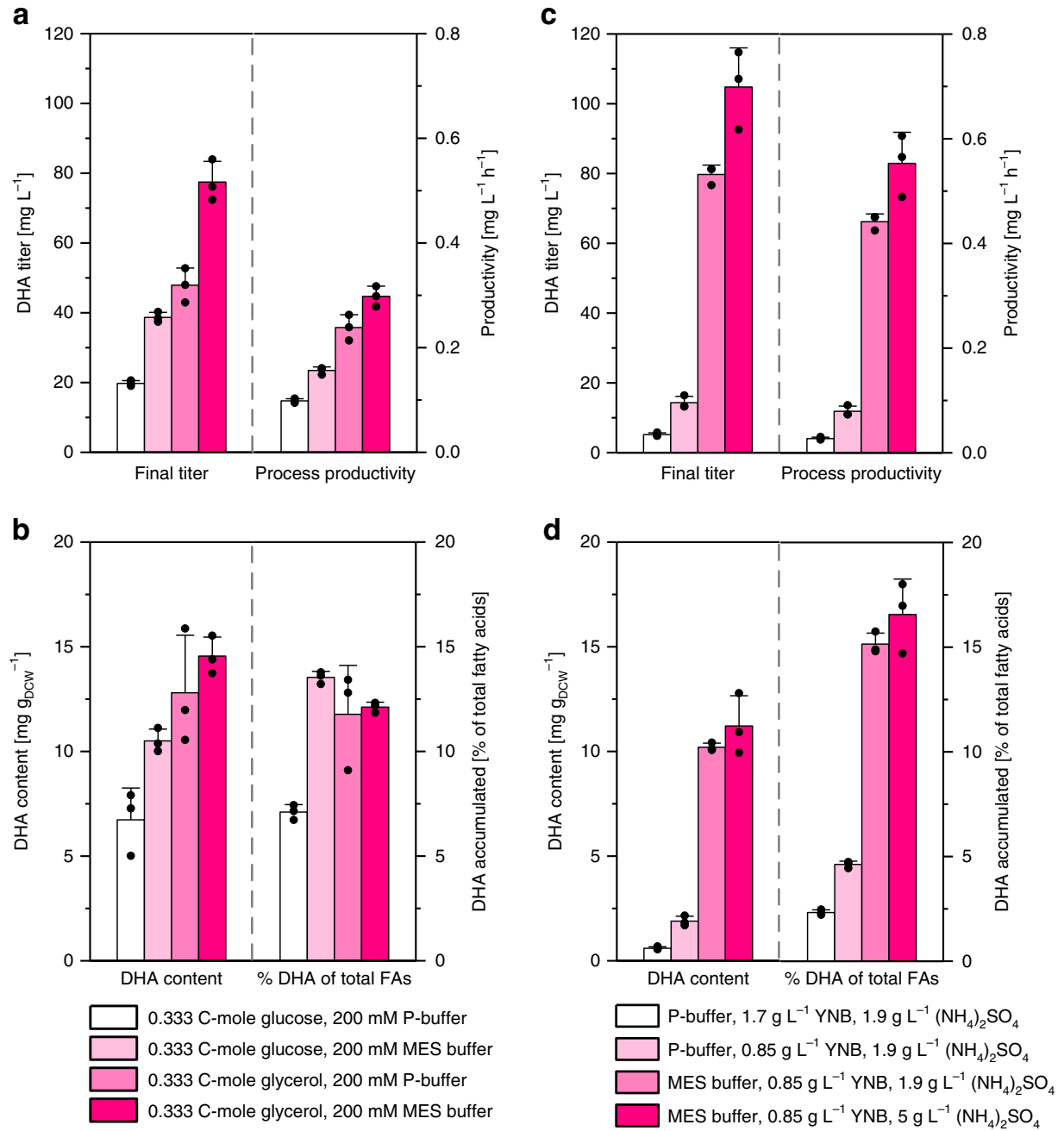

Fig. 4 DHA production improvement in Y. lipolytica Po1h::Af4 based on an optimization of medium composition. Shake flask cultivations were carried out in triplicates. Performance parameters were determined after $200 \mathrm{~h}$ of cultivation. Graphs show means and s.d. Source data are provided as a Source Data file

led to further extension of the LC-PUFA product spectrum upon heterologous expression in Y. lipolytica. In particular, a Y. lipolytica strain producing decent amounts of $n-3$ DPA (48 mg L-1/ $5.7 \%$ of TFAs under non-optimized fermentation conditions) was created. This long-chain $\omega-3$ fatty acid was shown to be very beneficial for human health ${ }^{26}$, but its concentration in marine fish is substantially lower than those of EPA and $\mathrm{DHA}^{25}$.

With regard to further development of the LC-PUFA production system established in this study, it will be mandatory to significantly increase the production titers of the LC-PUFAs of interest to reach a cost-effective commercial production. In order to do so, the fermentation procedure has to be further optimized in the matter of media, temperature, duration, and so on, and the amount and the productivity of the (hybrid) PUFA synthases have to be enhanced in vivo. Besides, the genomes of the transgenic Y. lipolytica strains can be modified with respect to inactivation or overexpression of genes, which are relevant for production (systems metabolic engineering). As a result, LCPUFAs should be enriched in the triacylglycerol fraction of the cell and thus increasingly accumulated in intracellular lipid droplets. On the other hand, an adequate supply of the PUFA synthase with the substrates malonyl-CoA and NADPH can be ensured and potential PUFA-degrading reactions can be reduced. 

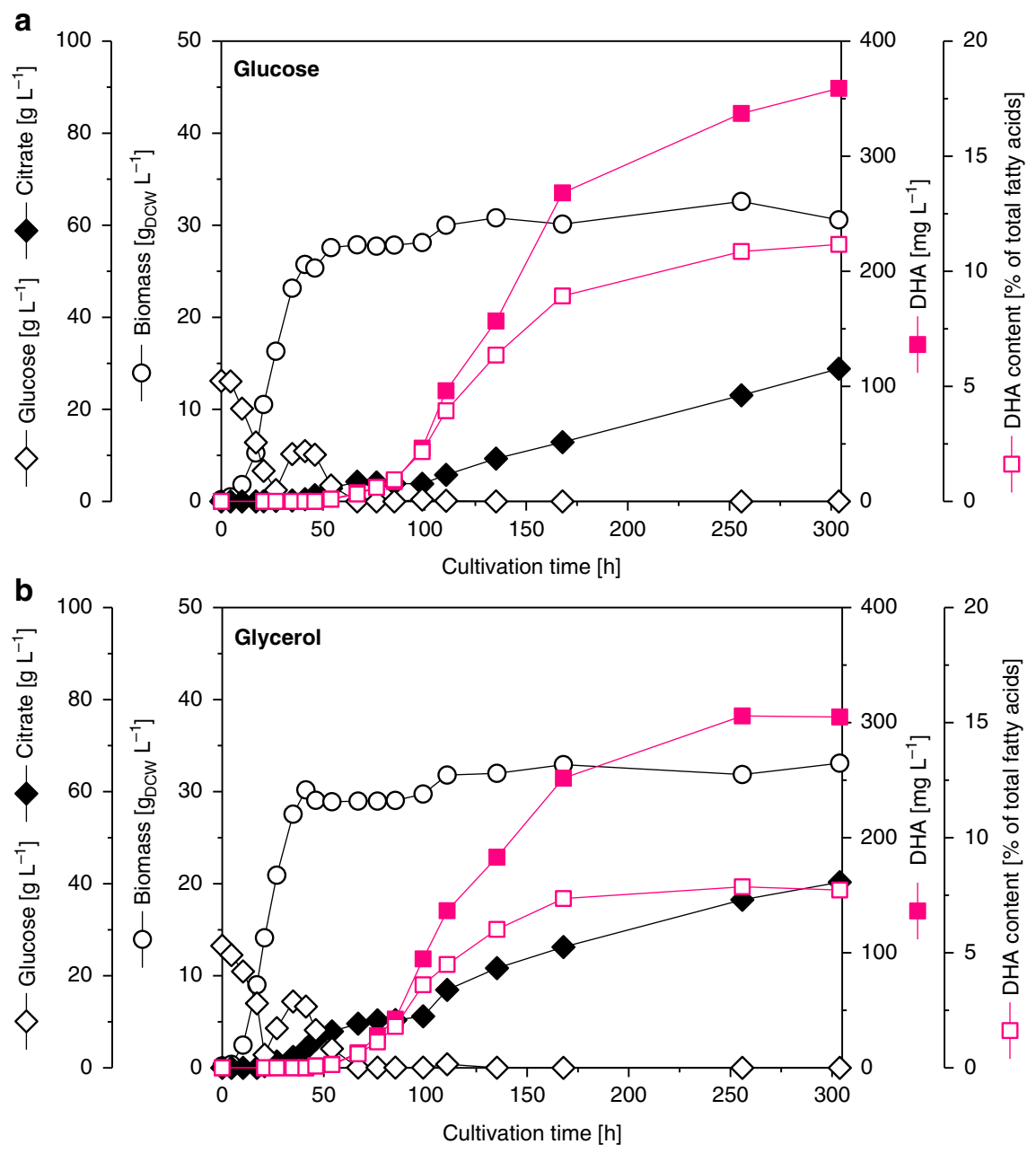

Fig. 5 DHA production in a fed-batch process. The designed producer Y. lipolytica Po1h::Af4 was grown in minimal medium on either glucose or glycerol as sole carbon source. Fermentation was conducted at $28^{\circ} \mathrm{C}, \mathrm{pH} 5.5$, and a dissolved oxygen level of $5 \%$ during the feed phase. Substrate feeding was initiated upon carbon depletion. Coefficients of variation (CVs) across biological replicates were below $5 \%$ for biomass, substrate, and citrate levels, and below $10 \%$ for PUFA and native fatty acid content. Source data are provided as a Source Data file

As Y. lipolytica has turned out to be an especially suitable microbe for LC-PUFA production, not least because of the ability to accumulate large amounts of lipids ${ }^{6}$, industrial efforts from DuPont (USA) on the engineering of Y. lipolytica strains capable of producing large amounts of LC-PUFAs were also reported ${ }^{12,13}$. Contrary to the Y. lipolytica strains generated in this study, which employ multifunctional PUFA synthases, DuPont made use of alternatingly acting position-specific desaturases and elongases, belonging to the aerobic PUFA biosynthetic pathway. In order to ensure efficient expression of the heterologous genes in Y. lipolytica, integration vectors with codon-optimized coding sequences driven by strong $Y$. lipolytica promoters were constructed. In total, three copies of $\Delta^{12}$ desaturase genes, two copies of $\Delta^{6}$ desaturase genes, four copies of $\mathrm{C}_{18 / 20}$ elongase genes, five copies of $\Delta^{5}$ desaturase genes, three copies of $\Delta^{17}$ desaturase genes, three copies of $\mathrm{C}_{16 / 18}$ elongase genes, one copy of a $\mathrm{C}_{20 / 22}$ elongase gene, and one copy of a $\Delta^{4}$ desaturase gene have been integrated into the genome of DuPont's $Y$. lipolytica strain Y $3000^{13}$. Moreover, the acyl-CoA oxidase 3 gene POX3 and an endogenous $\Delta^{12}$ desaturase had to be inactivated. In addition to the fatty acids produced by the corresponding wild type, strain Y3000 synthesizes 5.6\% DHA, 18.3\% n-3 DPA, 9.7\% $\mathrm{C}_{20}$ PUFAs, and $30.1 \%$ GLA of TFAs. As opposed to this, simply four biosynthetic genes encoding the DPA/DHA-type myxobacterial PUFA synthase and the PPTase from A. fasciculatus (SBSr002) were integrated into the genome of Y. lipolytica Polh. The resulting strain Y. lipolytica Polh::Af4 produces already 10.5\% DHA with high selectivity (production of only $0.4 \%$ nonpreferred PUFAs) under non-optimized fermentation conditions.

Substantial advantages of LC-PUFA biosynthesis via PUFA synthases as opposed to exploitation of the aerobic pathways are the independence from endogenous fatty acids as biosynthetic precursors and the lower consumption of $\mathrm{NAD}(\mathrm{P}) \mathrm{H}$. For instance, de novo synthesis of DHA catalyzed by PUFA synthases merely relies on $14 \mathrm{NADPH}$ molecules. However, using the aerobic route in which palmitic acid (synthesized by fatty acid synthase using $14 \mathrm{NADPH}$ molecules) is converted into DHA via diverse fatty acid desaturases and elongases, additionally $12 \mathrm{NAD}$ (P) H molecules are consumed. Therefore, the production of LCPUFAs using PUFA synthases described here should in the future allow for an optimized and highly efficient process towards these valuable compounds.

\section{Methods}

Sequence analysis and design of synthetic gene clusters. The sequences of the $p f a$ BGCs plus the genes encoding the PPTase from A. fasciculatus (SBSr002) and $M$. rosea (SBNa008) were analyzed and compared to the genome sequence of $Y$. lipolytica CLIB $122^{27}$ retrieved from NCBI Genome RefSeq NC_006067, NC_006068, NC_006069, NC_006070, NC_006071, and NC_006072. Based on this, relevant parameters for constructional and functional sequence design were defined to generate artificial pathway versions using the evoMAG ${ }^{\text {is }}$ software 
package (ATG:biosynthetics GmbH) ${ }^{19}$. The sequence design process included engineering of restriction sites, adaptation of the codon bias, and elimination of sequence repeats, as well as rare codon clusters, elimination of potential donor splice signals, and introduction of hidden stop codons in unused frames. Further details on the performed sequence analyses and the design of synthetic BGCs can be found in Supplementary Note 1.

Culture conditions. Escherichia coli $\mathrm{DH} 10 \mathrm{~B}^{28}$ and E. coli NEB 10- $\beta$ (New England Biolabs) were used for cloning experiments. Escherichia coli HS996/pSC101-BADgbaA $\left(\operatorname{tet}^{\mathrm{R}}\right)^{29}$ were used for modification of a plasmid using Red/ET recombination. The cells were grown in $2 \mathrm{xYT}$ medium (1.6\% tryptone, $1 \%$ yeast extract, $0.5 \%$ $\mathrm{NaCl})$, in $\mathrm{LB}$ medium or on LB agar $(1 \%$ tryptone, $0.5 \%$ yeast extract, $0.5 \% \mathrm{NaCl}$ $(1.5 \%$ agar) $)$ at $30-37^{\circ} \mathrm{C}$ (and $200 \mathrm{rpm}$ ) overnight. Antibiotics were used at the following concentrations: $50 \mu \mathrm{g} \mathrm{mL}^{-1}$ kanamycin, $34 \mu \mathrm{g} \mathrm{mL}^{-1}$ chloramphenicol, and $6 \mu \mathrm{g} \mathrm{mL}^{-1}$ tetracycline.

Auxotrophic Y. lipolytica strain Polh (CLIB 882) ${ }^{4}$ was obtained from Centre International de Ressources Microbiennes (CIRM)-Levures, Institut National de la Recherche Agronomique (INRA), AgroParisTech (Thiverval-Grignon, France). It was grown in YPD medium or on YPD agar containing $1 \%$ yeast extract, $2 \%$ peptone, and $2 \%$ dextrose. Prototrophic transformants were grown on minimal YNB- $\mathrm{N}_{5000}$ agar containing $0.67 \%$ yeast nitrogen base (with $75 \%\left(\mathrm{NH}_{4}\right)_{2} \mathrm{SO}_{4}$ and without amino acids), $1 \%$ glucose, and $1.5 \%$ agar or in minimal YNBG liquid medium containing $0.67 \%$ yeast nitrogen base (with $75 \%\left(\mathrm{NH}_{4}\right)_{2} \mathrm{SO}_{4}$ and without amino acids), $2 \%$ (w/v) glycerol, and $50 \mathrm{mM}$ potassium phosphate buffer, $\mathrm{pH} 6.8$. The cultures were incubated at $28-30^{\circ} \mathrm{C}$.

Isolation of genomic DNA from Y. lipolytica. Isolation of genomic DNA from yeast cells for genome sequencing was carried out using the method of Hoffman and Winston ${ }^{30}$. In the first step, $50 \mathrm{~mL}$ of a culture of $Y$. lipolytica grown at $28^{\circ} \mathrm{C}$ were harvested by centrifugation at $12,000 \times g$ for $5 \mathrm{~min}$. The supernatant was decanted, and $200 \mu \mathrm{L}$ lysis buffer $(2 \%(\mathrm{v} / \mathrm{v})$ Triton X-100, $1 \%$ sodium dodecyl sulfate, $100 \mathrm{mM}$ $\mathrm{NaCl}, 1 \mathrm{mM}$ EDTA, and $10 \mathrm{mM}$ Tris-Cl, $\mathrm{pH}$ 8.0), $200 \mu \mathrm{L}$ phenol:chloroform:isoamyl alcohol (25:24:1), plus $0.3 \mathrm{~g}$ acid-washed glass beads $(425-600 \mu \mathrm{m})$ were added to the cells. The tube was vortexed for 3 min. Afterwards, $200 \mu \mathrm{L}$ TE buffer $(10 \mathrm{mM}$ Tris-Cl, $\mathrm{pH}$ 8.0, and $1 \mathrm{mM}$ EDTA) were added, and the sample was centrifuged at $21,000 \times g$ for $5 \mathrm{~min}$. The upper aqueous phase was transferred to a tube containing $1 \mathrm{~mL}$ ice-cold ethanol and mixed by inversion. The sample was centrifuged at $21,000 \times g$ for $2 \mathrm{~min}$, and the supernatant was discarded. The pellet was resuspended in $400 \mu \mathrm{L}$ TE buffer plus $30 \mu \mathrm{g}$ RNase A. After incubation at $37^{\circ} \mathrm{C}$ for $15 \mathrm{~min}, 44 \mu \mathrm{L}$ of $4 \mathrm{M}$ ammonium acetate and $1 \mathrm{~mL}$ ice-cold ethanol were added, and the tube was inverted to mix. Genomic DNA was precipitated at $-80^{\circ} \mathrm{C}$ for $30 \mathrm{~min}$ to increase yield. The sample was centrifuged at $21,000 \times g$ for $2 \mathrm{~min}$, and the supernatant was discarded. The pellet was washed with $700 \mu \mathrm{L}$ of $70 \%$ ethanol, centrifuged at $21,000 \times g$ for $1 \mathrm{~min}$, and the supernatant was discarded. The dried DNA was resuspended in $50 \mu \mathrm{L}$ of $5 \mathrm{mM}$ Tris- $\mathrm{Cl}, \mathrm{pH} 8.0$

Transformation of $\mathbf{Y}$. lipolytica. Transformation of $Y$. lipolytica was carried out using a protocol developed by M.-T. Le Dall, modified by C. Madzak (Laboratoire de Microbiologie de l'Alimentation au Service de la Santé (MICALIS), AgroParisTech, Thiverval-Grignon, France; personal communication). One loopful of $Y$. lipolytica cells from a YPD agar plate grown at $30^{\circ} \mathrm{C}$ overnight were resuspended in $1 \mathrm{~mL}$ TE buffer in a sterile tube. The cells were centrifuged at $10,000 \times g$ for $1 \mathrm{~min}$, and the supernatant was discarded. After resuspension in $600 \mu \mathrm{L}$ of $0.1 \mathrm{M}$ lithium acetate, $\mathrm{pH} 6.0$, the cells were incubated at $28^{\circ} \mathrm{C}$ for $1 \mathrm{~h}$ in a water bath. The samples were centrifuged at $850 \times g$ for $2 \mathrm{~min}$, the supernatant was discarded, and the cells were softly resuspended in $80 \mu \mathrm{L}$ of $0.1 \mathrm{M}$ lithium acetate, $\mathrm{pH}$ 6.0. Forty microliters of competent cells were mixed with $2.5 \mu \mathrm{L}$ herring testes carrier DNA (10 $\mathrm{mg} \mathrm{mL}^{-1}$ in TE buffer, denatured) and $2.5 \mu \mathrm{L}$ linear DNA to be transferred. The samples were incubated at $28^{\circ} \mathrm{C}$ for $15 \mathrm{~min}$ in a water bath, and $350 \mu \mathrm{L}$ of $40 \%$ PEG 4000 in $0.1 \mathrm{M}$ lithium acetate, $\mathrm{pH} 6.0$ (plus $16 \mu \mathrm{L}$ of $1 \mathrm{M}$ dithiothreitol in case of non-targeted genome integration) were added. After incubation of the cells at $28^{\circ} \mathrm{C}$ for $1 \mathrm{~h}$ in a water bath, $40 \mu \mathrm{L}$ dimethyl sulfoxide (DMSO) were added. Subsequently, heat shock was carried out at $39^{\circ} \mathrm{C}$ for $10 \mathrm{~min}$ in a heating block. Six hundred microliters of $0.1 \mathrm{M}$ lithium acetate, $\mathrm{pH}$ 6.0, were then added, and the cells were plated onto YNB- $\mathrm{N}_{5000}$ agar. The plates were incubated at $30^{\circ} \mathrm{C}$ for 3 days. Thereafter, selected colonies were transferred in $1 \mathrm{~mL}$ YNBD or YNBG medium and cultivated at $28-30{ }^{\circ} \mathrm{C}$ and $900 \mathrm{rpm}$. The constructed strains are listed in Supplementary Table 13.

Construction of an initial plasmid for random integration. For modification of plasmid pINA1312 in order to construct plasmid pINA1312-SdaI-cm ${ }^{\mathrm{R}}-\mathrm{PacI}$ (Supplementary Table 12), a $1.2 \mathrm{~kb}$ DNA fragment containing a chloramphenicol resistance gene flanked with $S d a \mathrm{I}$ and $\mathrm{PacI}$ restriction sites as well as the appropriate homology arms was amplified from plasmid pACYC184 (New England Biolabs) using primers $\mathrm{HA}+\mathrm{SdaI}+\mathrm{cm}^{\mathrm{R}}$ for pINA1312_fwd and $\mathrm{HA}+\mathrm{PacI}+\mathrm{cm}^{\mathrm{R}}$ for pINA1292+1312_rev (Supplementary Table 11). PCR was performed with Taq DNA polymerase (Thermo Scientific) under standard conditions according to the manufacturer's protocol. The reactions contained $8 \%$ glycerol and were carried out in an Eppendorf Mastercycler under the following conditions: initial denaturation for $3 \mathrm{~min}$ at $95^{\circ} \mathrm{C} ; 30$ cycles consisting of denaturation for $30 \mathrm{~s}$ at $95^{\circ} \mathrm{C}$, annealing for $30 \mathrm{~s}$ at $62^{\circ} \mathrm{C}$, and extension for $1 \mathrm{~min} / \mathrm{kb}$ at $72^{\circ} \mathrm{C}$; and a final extension for 10 min at $72^{\circ} \mathrm{C}$. The linear plus circular homologous Red/ET recombineering ${ }^{14}$ was performed using $1.5 \mu \mathrm{L}$ of the PCR product.

Construction of plasmids for targeted integration. A cloning plasmid with chloramphenicol resistance gene, p15A origin of replication, and a multiple cloning site comprising SgsI, NotI, SgrDI, SdaI, PacI, and SwaI restriction sites was constructed. This plasmid ( $2.1 \mathrm{~kb})$ was amplified from plasmid pACYC184 using overlapping primers $\mathrm{cm}^{\mathrm{R}} S d a \mathrm{I}+P a c \mathrm{I}+S w a \mathrm{I}+S g s \mathrm{I}$ fwd and p15A_SgsI + NotI +SgrDI_rev. PCR was performed with the PCR extender system (5 Prime) under standard conditions according to the manufacturer's protocol. The reactions were carried out in an Eppendorf Mastercycler under the following conditions: initial denaturation for $2 \mathrm{~min}$ at $94^{\circ} \mathrm{C} ; 20$ cycles consisting of denaturation for $20 \mathrm{~s}$ at $94^{\circ} \mathrm{C}$, annealing for $20 \mathrm{~s}$ at $66^{\circ} \mathrm{C}$, and extension for $1 \mathrm{~min} \mathrm{~kb}{ }^{-1}$ at $72^{\circ} \mathrm{C}$; and a final extension for $10 \mathrm{~min}$ at $72^{\circ} \mathrm{C}$. The $h p h$ gene $(1 \mathrm{~kb})$ encoding the hygromycin $\mathrm{B}$ phosphotransferase from E. coli/Klebsiella pneumoniae was amplified from plasmid Tn5_epo_hyg (Y. Zhang, unpublished) using primers $h p h \_A \_f w d$ and $h p h \_B a m-$ HI_rev. PCR was performed with Phusion DNA polymerase (Thermo Scientific) under standard conditions according to the manufacturer's protocol. The reactions contained 5\% DMSO and were carried out in an Eppendorf Mastercycler under the following conditions: initial denaturation for $2 \mathrm{~min}$ at $98^{\circ} \mathrm{C} ; 30$ cycles consisting of denaturation for $15 \mathrm{~s}$ at $98^{\circ} \mathrm{C}$, annealing for $20 \mathrm{~s}$ at $70{ }^{\circ} \mathrm{C}$, and extension for $10 \mathrm{~s} \mathrm{~kb}^{-1}$ at $72{ }^{\circ} \mathrm{C}$; and a final extension for $10 \mathrm{~min}$ at $72^{\circ} \mathrm{C}$. The PCR amplicon was inserted into plasmid pINA1312 via PmlI and BamHI restriction sites, yielding plasmid pINA1312-hph. For the fusion of hp4d-hph and LIP2t, the strong hybrid promoter hp4 $4 \mathrm{~d}^{3}$ and $h p h$ plus the $5^{\prime}$ part of the LIP2 terminator as overlapping sequence were amplified as the first fragment $(1.6 \mathrm{~kb})$ from plasmid pINA1312-hph using primers UAS1B_SdaI_fwd and $h p h+L I P 2 \mathrm{t}$ overlap_rev. The second fragment $(0.1 \mathrm{~kb})$ contained the LIP2 terminator from Y. lipolytica plus the $3^{\prime}$ end of $h p h$ as overlapping sequence and was amplified from plasmid pACYC_BB1-4_C1_V1 using primers LIP2t $+h p h$ overlap_fwd and LIP2t_SgrDI_rev. PCR amplification of the two fragments to be spliced was performed with Phusion DNA polymerase (Thermo Scientific) under standard conditions according to the manufacturer's protocol. The reactions contained 5\% DMSO and were carried out in an Eppendorf Mastercycler under the following conditions: initial denaturation for $2 \mathrm{~min}$ at $98^{\circ} \mathrm{C} ; 20$ cycles consisting of denaturation for $15 \mathrm{~s}$ at $98^{\circ} \mathrm{C}$, annealing for $20 \mathrm{~s}$ at $64^{\circ} \mathrm{C}$, and extension for $10 \mathrm{skb}^{-1}$ at $72^{\circ} \mathrm{C}$; and a final extension for $10 \mathrm{~min}$ at $72^{\circ} \mathrm{C}$. For the subsequent overlap extension PCR using primers UAS1B_SdaI_fwd and LIP2t_SgrDI_rev, the two amplified fragments were used as templates. PCR of the $1.7 \mathrm{~kb}$ fragment was performed as described for the amplification of the two fragments to be spliced. Plasmid pKG1 was constructed by ligation of hp4d-hph-LIP2t with the chloramphenicol resistance gene, p15A origin of replication, and the multiple cloning site via $S d a I$ and $S g r D I$ restriction sites. One kilobase homology regions upstream and downstream of the preferred integration site were amplified from genomic DNA of $Y$. lipolytica Polh::Af2 clone C using primers PIS_fragment1_NotI_fwd/PIS_ fragment1_PciI+SgrDI_rev and PIS_fragment2_PacI_fwd/PIS_fragment2_SwaI_rev. PCR was performed with Phusion DNA polymerase (Thermo Scientific) under standard conditions according to the manufacturer's protocol. The reactions contained 5\% DMSO and were carried out in an Eppendorf Mastercycler under the following conditions: initial denaturation for $2 \mathrm{~min}$ at $98^{\circ} \mathrm{C} ; 20$ cycles consisting of denaturation for $15 \mathrm{~s}$ at $98^{\circ} \mathrm{C}$, annealing for $20 \mathrm{~s}$ at $61{ }^{\circ} \mathrm{C}$, and extension for $10 \mathrm{~s} \mathrm{~kb}^{-1}$ at $72^{\circ} \mathrm{C}$; and a final extension for $10 \mathrm{~min}$ at $72^{\circ} \mathrm{C}$. The PCR amplicons were inserted into plasmid pKG1 via NotI/SgrDI and PacI/SwaI restriction sites, yielding plasmid pKG1-PIS. In order to generate plasmid pKG2-PIS, hp4d-hph-LIP2t of plasmid pKG1-PIS was replaced by the orotidine 5 -phosphate decarboxylase expression cassette from Y. lipolytica (URA3p-URA3-URA3t) located on plasmid pINA1312$S d a \mathrm{I}-\mathrm{cm}^{\mathrm{R}}-\mathrm{PacI}$ via $P c i \mathrm{I}$ and $S d a \mathrm{I}$ restriction sites. Sequences of primers used in this study are listed in Supplementary Table 11 and further information on the plasmids constructed in this study are provided in Supplementary Table 12.

Cloning of expression plasmids containing synthetic pfa BGCs. For the heterologous expression of artificial PUFA biosynthetic pathways in $Y$. lipolytica, four building blocks containing the three $p f a$ genes as well as gene $p p t$ encoding the PPTase originating from A. fasciculatus (SBSr002) $(\rightarrow$ synthetic BGCs version $\mathrm{C} 1 \_\mathrm{V} 1$ and $\left.\mathrm{C} 1 \_\mathrm{V} 2\right)$ and six building blocks containing the three $p f a$ genes as well as gene ppt encoding the PPTase originated from $M$. rosea (SBNa008) ( $\rightarrow$ synthetic BGC version C3) were designed and supplied by gene synthesis companies in the standard cloning vectors $\mathrm{pBSK}$ or $\mathrm{pGH}$. Each coding sequence is flanked by the strong hybrid hp4d promoter ${ }^{3}$ plus LIP2 terminator for Y. lipolytica. Moreover, non-coding sequences were attached to each gene allowing for the connection of the transcription units by $200 \mathrm{bp}$ intergenic linkers. In the course of constructional design, the unique SdaI, ApaLI, NcoI, SalI, AclI, KpnI, FseI, AvrII, and PacI restriction sites were introduced for cloning purposes as well as for exchangeability of genes/domains and were excluded from any other unwanted position within the BGC.

A cloning plasmid with kanamycin resistance gene, p15A origin of replication, and restriction sites for assembly of the four DNA building blocks was constructed. Therefore, a multiple cloning site comprising DraI, SdaI, ApaLI, NcoI, SalI, AclI, AatII, NgoMIV, AvrII, PacI, and BamHI restriction sites $(0.1 \mathrm{~kb})$ was amplified by 
PCR using overlapping primers MCS for pACYC_fwd and MCS for pACYC_rev. PCR was performed with Phusion DNA polymerase (Thermo Scientific) under standard conditions according to the manufacturer's protocol. The reactions contained 5\% DMSO and were carried out in an Eppendorf Mastercycler under the following conditions: initial denaturation for $2 \mathrm{~min}$ at $98^{\circ} \mathrm{C} ; 30$ cycles consisting of denaturation for $15 \mathrm{~s}$ at $98^{\circ} \mathrm{C}$, annealing for $20 \mathrm{~s}$ at $72^{\circ} \mathrm{C}$, and extension for $10 \mathrm{~s} \mathrm{~kb}^{-1}$ at $72{ }^{\circ} \mathrm{C}$; and a final extension for $10 \mathrm{~min}$ at $72^{\circ} \mathrm{C}$. The MCS was inserted into plasmid pACYC177 via DraI and BamHI restriction sites, yielding plasmid pACYC_assembly.

The four building blocks of synthetic BGC version C1_V1 were stitched together in plasmid pACYC_assembly: building block 1 was inserted into pACYC_assembly via $S d a \mathrm{I}$ and $A p a \mathrm{LI}$ restriction sites, generating plasmid pACYC_BB1_Cl_V1; building block 4 was inserted into pACYC_BB1_C1_V1 via AvrII and PacI restriction sites, generating plasmid pACYC_BB1+4_C1_V1; building block 2 was inserted into pACYC_BB1+4_C1_V1 via ApaLI and Acll restriction sites, generating plasmid pACYC_BB1+2+4_C1_V1; building block 3 was inserted into pACYC_BB1+2 +4_C1_V1 via AclI and AvrII restriction sites, generating plasmid pACYC_BB14_C1_V1. The backbone of plasmid pACYC_BB1-4_C1_V1 was exchanged for the backbone of plasmid pINA1312-SdaI-cm ${ }^{\mathrm{R}}-\mathrm{PacI}$ via $\mathrm{S} d a \mathrm{I}$ and $\mathrm{PacI}$ restriction sites, yielding plasmid pAf2. Plasmid pAf4 was constructed for targeted genome integration by exchange of the backbone of plasmid pAf2 for the backbone of plasmid pKG2-PIS via $S d a \mathrm{I}$ and $\mathrm{PacI}$ restriction sites.

The six building blocks of synthetic BGC version C3 were stitched together in plasmids pACYC_assembly and pAf2: building block 1 was inserted into pACYC_assembly via SdaI and ApaLI restriction sites, generating plasmid pACYC_BB1_C3; building block 2 was inserted into pACYC_BB1_C3 via ApaLI and $\mathrm{NcoI}$ restriction sites, generating plasmid pACYC_BB1+2_C3; building block 3 was inserted into pACYC_BB1+2_C3 via NcoI and $\mathrm{Acl}$ I restriction sites, generating plasmid pACYC_BB1-3_C3; building blocks 1-3 of pACYC_BB1-3_C3 were inserted into pAf2 via $S d a I$ and $A c l$ restriction sites, generating plasmid pSyn_BB1-3_C3; building block 4 was inserted into pSyn_BB1-3_C3 via AclI and $K p n \mathrm{I}$ restriction sites, generating plasmid pSyn_BB1-4_C3; building block 5 was inserted into pSyn_BB1-4_C3 via KpnI and AvrII restriction sites, generating plasmid pSyn_BB1-5_C3; building block 6 was inserted into pSyn_BB1-5_C3 via AvrII and PacI restriction sites, generating plasmid pSyn_BB1-6_C3. The backbone of plasmid pSyn_BB1-6_C3 was exchanged for the backbone of plasmid pACYC_assembly or for the backbone of plasmid pKG2-PIS via SdaI and PacI restriction sites, yielding either plasmid pACYC_BB1-6_C3 or plasmid pMr1. Sequences of primers used in this study are listed in Supplementary Table 11 and further information on the plasmids constructed in this study are provided in Supplementary Table 12.

For the heterologous expression of the artificial PUFA biosynthetic pathway originating from $M$. rosea (SBNa008) with improved $5^{\prime}$ coding sequences in $Y$. lipolytica, the existing plasmid pACYC_BB1-6_C3 was modified in the following way: building block 6 was exchanged for BB6_C3_mod 5' via AvrII and PacI restriction sites, generating plasmid pACYC_BB1-6_C3_BB6_mod 5'; the 5' part of building block 2 of pACYC_BB1-6_C3_BB6_mod 5' was exchanged for BB2_C3_mod 5' via ApaLI and PciI restriction sites, generating plasmid pACYC_BB1-6_C3_BB2+6_mod 5'; the 5' part of building block 4 of pACYC_BB1-6_C3_BB2+6_mod 5' was exchanged for BB4_C3_mod 5' via AclI and $K p n I$ restriction sites, generating plasmid pACYC_BB1-6_C3_BB2+4+6_mod $5^{\prime}$; building block 1 of pACYC_BB1-6_C3_BB2+4+6_mod 5' was exchanged for BB1_C3_mod 5' via SdaI and ApaLI restriction sites, generating plasmid pACYC_BB1-6_C3_BB1+2+4+6_mod 5' (Supplementary Table 12). The backbone of plasmid pACYC_BB1-6_C3_BB1+2+4+6_mod 5' was exchanged for the backbone of plasmid pKG2-PIS via SdaI and PacI restriction sites, yielding plasmid pMr2 (Supplementary Table 12).

Cloning of expression plasmids containing chimeric pfa BGCs. For the heterologous expression of artificial hybrid PUFA biosynthetic pathways in Y. lipolytica, several chimeric PUFA expression constructs were generated. Plasmid pACYC_SynHyb1 was generated by replacement of hp4d, the KS2, the CLF, the $\mathrm{AT} 2$, the $\mathrm{DH} 2$, and the $\mathrm{DH} 3$ domain of synthetic gene $p f a 3$ originating from $A$. fasciculatus (SBSr002) located on plasmid PACYC_BB1-4_C1_V1 by the homologous domains of synthetic gene pfa 3 originating from $M$. rosea (SBNa008) located on plasmid pSyn_BB1-6_C3 via AclI and FseI restriction sites. The backbone of plasmid pACYC_SynHyb1 was exchanged for the backbone of plasmid pKG2-PIS via SdaI and PacI restriction sites, yielding plasmid pHyb2. The DH2, the DH3, and the AGPAT domain of synthetic chimeric gene $p f a 3$ located on plasmid $\mathrm{pHyb} 2$ were replaced by the homologous domains plus the DH4 domain of synthetic gene $p f a 3$ originating from $M$. rosea (SBNa008) located on plasmid pACYC_BB1-6_C3 via KpnI and AvrII restriction sites, generating plasmid pHyb1. Plasmid pHyb2a was generated by replacing the AGPAT domain of synthetic chimeric gene $p f a 3$ located on plasmid pHyb2 by a synthetic DNA fragment, consisting of the DH4 domain of synthetic gene pfa3 from M. rosea (SBNa008) plus the AGPAT domain of synthetic gene $p f a 3$ originating from A. fasciculatus (SBSr002), via FseI and AvrII restriction sites. Plasmid pHyb5 was generated by replacing the $\mathrm{DH} 2$ and the $\mathrm{DH} 3$ domain of synthetic gene $p f a 3$ originating from $A$. fasciculatus (SBSr002) located on plasmid pAf4 by the homologous domains of synthetic gene $p f a 3$ originating from $M$. rosea (SBNa008) located on plasmid
pACYC_BB1-6_C3 via KpnI and FseI restriction sites. Plasmid pHyb5a was generated by replacing the AGPAT domain of synthetic gene $p f a 3$ located on plasmid pHyb5 by a synthetic DNA fragment, consisting of the DH4 domain of synthetic gene $p f a 3$ from $M$. rosea (SBNa008) plus the AGPAT domain of synthetic gene pfa3 originating from A. fasciculatus (SBSr002), via FseI and AvrII restriction sites. The AGPAT domain of synthetic gene $p f a 3$ originating from A. fasciculatus (SBSr002) located on plasmid pAf4 was replaced by the DH4 domain plus the AGPAT domain of synthetic gene pfa3 originating from M. rosea (SBNa008) located on plasmid pACYC_BB1-6_C3 via FseI and AvrII, yielding plasmid pHyb6. The AGPAT domain of synthetic gene pfa3 located on plasmid pHyb6 was replaced by a synthetic DNA fragment consisting of the DH4 domain of synthetic gene $p f a 3$ from M. rosea (SBNa008) plus the AGPAT domain of synthetic gene pfa3 originating from A. fasciculatus (SBSr002), via FseI and AvrII restriction sites, yielding plasmid pHyb6b. In order to inactivate the DH4 domain of plasmid pHyb6b by an active site H2270A point mutation, the DH4 and the AGPAT domain were amplified in two fragments from plasmid pHyb6b using primers H2270A_fwd_2/H2270A_CA to GC_rev and H2270A_CA to GC_fwd/H2270_rev. The first fragment $(0.2 \mathrm{~kb})$ carried the CA to GC nucleotide exchanges at the $3^{\prime}$ end, whereas the second fragment $(2.2 \mathrm{~kb})$ carried these base exchanges at the $5^{\prime}$ end. PCR amplification of the two fragments to be spliced was performed with Phusion DNA polymerase (Thermo Scientific) under standard conditions according to the manufacturer's protocol. The reactions contained 5\% DMSO and were carried out in an Eppendorf Mastercycler under the following conditions: initial denaturation for $2 \mathrm{~min}$ at $98^{\circ} \mathrm{C} ; 20$ cycles consisting of denaturation for $15 \mathrm{~s}$ at $98^{\circ} \mathrm{C}$, annealing for $20 \mathrm{~s}$ at $72{ }^{\circ} \mathrm{C}$, and extension for $10 \mathrm{skb}^{-1}$ at $72^{\circ} \mathrm{C}$; and a final extension for $10 \mathrm{~min}$ at $72^{\circ} \mathrm{C}$. For the subsequent overlap extension PCR using primers H2270A_fwd_2 and H2270A_rev, the two amplified fragments were used as templates. PCR of the $2.4 \mathrm{~kb}$ fragment was performed as described for the amplification of the two fragments to be spliced. In order to generate plasmid pHyb6b-H2270A, the DH4 and the AGPAT domain of plasmid pHyb6b were replaced by the PCR amplicon via FseI and AvrII restriction sites. Plasmid pHyb7 was generated by replacing synthetic genes $p f a 2$ and $p f a 3$ originating from $A$. fasciculatus (SBSr002) located on plasmid pAf4 by synthetic genes $p f a 2$ and $p f a 3$ originating from $M$. rosea (SBNa008) located on plasmid pACYC_BB1-6_C3 via ApaLI and AvrII restriction sites. Plasmid pACYC_SynHyb3 was generated by replacement of synthetic gene $p f a 2$ originating from A. fasciculatus (SBSr002) located on plasmid pACYC_BB1-4_C1_V1 by synthetic gene $p f a 2$ originating from M. rosea (SBNa008) located on plasmid pMr1 via ApaLI and Acll restriction sites The backbone of plasmid pACYC_SynHyb3 was exchanged for the backbone of plasmid pKG2-PIS via SdaI and PacI restriction sites, yielding plasmid $\mathrm{pHyb} 8$. The $\mathrm{DH} 1$ domain of synthetic gene $p f a 2$ originating from M. rosea (SBNa008) $(1.2 \mathrm{~kb})$ was amplified from plasmid pACYC_BB1-6_C3 using primers DH1-Mr_Kfl_fwd and linker 2_rev. PCR was performed with Phusion DNA polymerase (Thermo Scientific) under standard conditions according to the manufacturer's protocol. The reactions contained 5\% DMSO and were carried out in an Eppendorf Mastercycler under the following conditions: initial denaturation for $2 \mathrm{~min}$ at $98^{\circ} \mathrm{C} ; 20$ cycles consisting of denaturation for $15 \mathrm{~s}$ at $98^{\circ} \mathrm{C}$, annealing for $20 \mathrm{~s}$ at $66^{\circ} \mathrm{C}$, and extension for $10 \mathrm{~s} \mathrm{~kb}^{-1}$ at $72^{\circ} \mathrm{C}$; and a final extension for $10 \mathrm{~min}$ at $72^{\circ} \mathrm{C}$. In order to generate plasmid pACYC_SynHyb4, the DH1 domain of synthetic gene $p f a 2$ originating from $A$. fasciculatus (SBSr002) located on plasmid pACYC_BB14_C1_V1 was replaced by the PCR amplicon via KfII and AclI restriction sites. The backbone of plasmid pACYC_SynHyb4 was exchanged for the backbone of plasmid pKG2-PIS via SdaI and PacI restriction sites, yielding plasmid pHyb9. For the fusion of the KR domain of synthetic gene $p f a 2$ originating from $M$. rosea (SBNa008) and the DH1 domain of synthetic gene $p f a 2$ originating from A. fasciculatus (SBSr002), the KR domain was amplified as the first fragment $(1.2 \mathrm{~kb})$ from plasmid pMr2 using primers KR-Mr_fwd and KR-Mr+DH1-Af overlap_rev. The second fragment $(1.1 \mathrm{~kb})$ contained the DH1 domain and was amplified from plasmid pAf4 using primers DH1-Af + KR-Mr overlap_fwd and linker 2_rev. PCR amplification of the two fragments to be spliced was performed with Phusion DNA polymerase (Thermo Scientific) under standard conditions according to the manufacturer's protocol. The reactions contained 5\% DMSO and were carried out in an Eppendorf Mastercycler under the following conditions: initial denaturation for $2 \mathrm{~min}$ at $98^{\circ} \mathrm{C} ; 20$ cycles consisting of denaturation for $15 \mathrm{~s}$ at $98^{\circ} \mathrm{C}$, annealing for $20 \mathrm{~s}$ at $67^{\circ} \mathrm{C}$, and extension for $10 \mathrm{~s} \mathrm{~kb}^{-1}$ at $72^{\circ} \mathrm{C}$; and a final extension for 10 min at $72^{\circ} \mathrm{C}$. For the subsequent overlap extension PCR using primers KR-Mr_fwd and linker 2_rev, the two amplified fragments were used as templates. PCR of the $2.2 \mathrm{~kb}$ fragment was performed as described for the amplification of the two fragments to be spliced. In order to generate plasmid pACYC_SynHyb7, the KR and the $\mathrm{DH} 1$ domain of synthetic gene $p f a 2$ originating from $M$. rosea (SBNa008) located on plasmid pACYC_BB1-6_C3_BB1+2+4+6_mod 5' was replaced by the PCR amplicon via SalI and AclI restriction sites. The backbone of plasmid pACYC_SynHyb7 was exchanged for the backbone of plasmid pKG2-PIS via SdaI and PacI restriction sites, yielding plasmid $\mathrm{pHyb} 15$. Sequences of primers used in this study are listed in Supplementary Table 11 and further information on the plasmids constructed in this study are provided in Supplementary Table 12.

Standard LC-PUFA production. For heterologous LC-PUFA production in $Y$. lipolytica, cultivations were carried out in $10 \mathrm{~mL}$ YNBG medium in $50 \mathrm{~mL}$ shake flasks. The medium was inoculated with an overnight culture (1:100) and incubated 
at $28^{\circ} \mathrm{C}$ for $168 \mathrm{~h}$. Afterwards, $1 \mathrm{~mL}$ of the culture was subjected to FAME extraction (see below)

Medium optimization for enhanced LC-PUFA production. In a number of studies, the composition of the YNBG production medium was varied to investigate the impact of different nutrients on LC-PUFA production. The experiments included (i) a variation of the primary carbon source (glucose, glycerol, acetate), (ii) a variation of the nitrogen source $\left(\mathrm{NH}_{4} \mathrm{Cl},\left(\mathrm{NH}_{4}\right)_{2} \mathrm{SO}_{4}\right)$, (iii) a variation of the $\mathrm{C} / \mathrm{N}$ ratio in the range of 4-55), and (iv) a variation of phosphate level by an adjustment of the YNB concentration (0.85-1.7 $\left.\mathrm{g} \mathrm{L}^{-1}\right)$, and a replacement of potassium phosphate buffer by MES in a range of $50-200 \mathrm{mM}$ ). Cultures were grown at $28^{\circ} \mathrm{C}$ in $500 \mathrm{~mL}$ baffled shake flasks with $50 \mathrm{~mL}$ medium on an orbital shaker $(230 \mathrm{rpm})$.

DHA production in a fed-batch process. The production performance of the DHA producer Y. lipolytica Polh::Af4 was evaluated in a fed-batch process. Fermentation was carried out in glucose and in glycerol-based medium using $1 \mathrm{~L}$ DASGIP bioreactors (Eppendorf, Jülich, Germany). The initial batch medium (300 $\mathrm{mL})$ contained per liter: $25 \mathrm{~g}$ glucose or $25.6 \mathrm{~g}$ glycerol, $5 \mathrm{~g}\left(\mathrm{NH}_{4}\right)_{2} \mathrm{SO}_{4}(\mathrm{C} / \mathrm{N}$ ratio 11), $1.7 \mathrm{~g}$ YNB w/o amino acids, $1 \mathrm{~g} \mathrm{KH}_{2} \mathrm{PO}_{4}, 200 \mathrm{mmol} \mathrm{MES}$ ( $\mathrm{pH} 5.5$ ), and $1 \mathrm{~mL}$ antifoam (Antifoam 204, Sigma, Germany). The process was inoculated with exponentially growing cells from an overnight pre-culture. Feeding $\left(600 \mathrm{~g} \mathrm{~L}^{-1}\right.$ glucose or $600 \mathrm{~g} \mathrm{~L}^{-1}$ glycerol) was started at a rate of $1.5 \mathrm{~mL} \mathrm{~h}^{-1}$, when the substrate was depleted. During the feed phase, the level of the carbon source was monitored at-line. The data were used to re-adjust the feed rate in order to avoid a limitation of the carbon source. Cultivation temperature was maintained constant at $28^{\circ} \mathrm{C}$. The $\mathrm{pH}$ and the $\mathrm{pO}_{2}$ level were monitored online with a $\mathrm{pH}$ electrode (Mettler Toledo, Gießen, Germany) and a $\mathrm{pO}_{2}$ electrode (Hamilton, Höchst, Germany). The $\mathrm{pH}$ was kept constant at $5.5 \pm 0.05$ by automated addition of $6 \mathrm{M}$ $\mathrm{NaOH}$ and $6 \mathrm{M} \mathrm{HCl}$. The dissolved oxygen level was maintained above $30 \%$ of saturation during the batch phase and was reduced to $5 \%$ during the feed phase by variation of stirrer speed and aeration rate. Data acquisition and process operations were controlled by the DASGIP control software 4.0 (Eppendorf, Jülich, Germany).

Quantification of extracellular metabolites. Glucose in culture supernatant was determined with a biochemical analyzer (YSI 2900, Kreienbaum, Germany). Sugar alcohols and organic acids were quantified by high-performance liquid chromatography (Agilent 1200 series, Waldbronn, Germany) equipped with a ultraviolet and refraction index detector using an Aminex HPX-87H column (Bio-Rad, Hercules, USA) at $45^{\circ} \mathrm{C}$ and isocratic elution with $12 \mathrm{mM} \mathrm{H}_{2} \mathrm{SO}_{4}$ at a flow rate of $0.5 \mathrm{~mL} \mathrm{~min}^{-1}$. Ammonium was quantified using a photometric kit (Spectroquant Ammonium Test, Merck, Darmstadt, Germany)

Preparation of FAMEs. The cells were harvested by centrifugation at $12,000 \times g$ for $5 \mathrm{~min}$, transferred to a glass vial, and dried in a vacuum concentrator. Subsequently, the CDW was determined. The cellular fatty acids were extracted using the FAME method ${ }^{31}$. For this purpose, the cells were transferred to a glass vial and dried in a vacuum concentrator. In all, $25-50 \mu \mathrm{g}$ of $n-3$ heneicosapentaenoic acid (HPA; 22:5) methyl ester (Cayman Chemical) or $n$-3 DPA methyl ester (SigmaAldrich) and $250-500 \mu \mathrm{L}$ of a mixture of methanol, toluene, and sulfuric acid $(50: 50: 2, \mathrm{v} / \mathrm{v} / \mathrm{v})$ were added. The vial was capped with a Teflon-lined screw cap and incubated at $80^{\circ} \mathrm{C}$ overnight. After the mixture was cooled to room temperature, $200-400 \mu \mathrm{L}$ of an aqueous solution consisting of $0.5 \mathrm{M} \mathrm{NH}_{4} \mathrm{HCO}_{3}$ and $2 \mathrm{M} \mathrm{KCl}$ were added, and the sample was vortexed for $30 \mathrm{~s}$. Phase separation was achieved by centrifugation at $4000 \times g$ for $5 \mathrm{~min}$ at room temperature. Seventy-five microliters of the upper phase were mixed with $25 \mu \mathrm{L} \mathrm{N}$-methyl- $N$-(trimethylsilyl)trifluoroacetamide and incubated at $37^{\circ} \mathrm{C}$ for $30 \mathrm{~min}$. Subsequently, the sample was used for GC-MS analysis.

Extraction and fractionation of lipids. Extraction of lipids from microbial cells was carried out using the method of Bligh and Dyer ${ }^{32}$, modified by Lewis et al. ${ }^{33}$, on a small scale. In the first step, the cell pellet from a $15 \mathrm{~mL}$ culture was transferred into a polypropylene tube. Successively, $4 \mathrm{~mL}$ chloroform, $8 \mathrm{~mL}$ methanol, and $3.2 \mathrm{~mL}$ of $1 \% \mathrm{NaCl}$ were added, and the tube was vortexed at high speed for 15 $\mathrm{s}$ after every addition. The sample was agitated on a tube rotator at $30 \mathrm{rpm}$ overnight. Four microliters of chloroform and $4 \mathrm{~mL}$ of $1 \% \mathrm{NaCl}$ were then added, and the tube was inverted 30 times. Phase separation was achieved by centrifugation at $4000 \times g$ for $5 \mathrm{~min}$ at room temperature. The bottom layer containing the lipid extract was evaporated to dryness under a gentle stream of nitrogen and dissolved in $1 \mathrm{~mL}$ of a chloroform:methanol mixture $(2: 1, \mathrm{v} / \mathrm{v})$. The lipid solution was fractionated by solid-phase extraction on a Strata SI-1 silica column with 100 $\mathrm{mg}$ sorbent mass (Phenomenex) equilibrated with chloroform $+1 \%$ acetic acid. Neutral lipids and free fatty acids were eluted from the column with $1 \mathrm{~mL}$ of chloroform $+1 \%$ acetic acid, glycolipids were eluted with $1.5 \mathrm{~mL}$ of an acetone: methanol mixture $(9: 1, \mathrm{v} / \mathrm{v})$, and phospholipids were eluted with $1 \mathrm{~mL}$ methanol. For GC-MS analysis, the fractions were dried in a vacuum concentrator and further processed according to the FAME method.
Analysis of FAMEs by GC-MS. GC-MS was carried out on an Agilent $6890 \mathrm{~N}$ gas chromatograph (Agilent Technologies) equipped with a 7683B split/splitless injector with autosampler (Agilent Technologies) and coupled to a 5973 electron impact mass-selective detector (Agilent Technologies). Helium was used as the carrier gas at a flow rate of $1 \mathrm{~mL} \mathrm{~min}^{-1}$. A measure of $0.2-5 \mu \mathrm{L}$ of the sample were injected in split mode (split ratio, 10:1). The analytical column was a (5\% phenyl)methylpolysiloxane capillary column (Agilent J\&W DB-5ht; $30 \mathrm{~m} \times 0.25 \mathrm{~mm}$ i.d. $\times$ $0.1 \mu \mathrm{m}$ film thickness, maximum temperature $400^{\circ} \mathrm{C}$; Agilent Technologies). The column temperature was kept at $130^{\circ} \mathrm{C}$ for $2.5 \mathrm{~min}$, increased to $240^{\circ} \mathrm{C}$ at a rate of $5^{\circ} \mathrm{C} \mathrm{min}-1$, then ramped to $300^{\circ} \mathrm{C}$ at $30{ }^{\circ} \mathrm{C} \mathrm{min}-1$, and held at $300^{\circ} \mathrm{C}$ for $5 \mathrm{~min}$. Other temperatures were as follows: inlet, $275^{\circ} \mathrm{C}$; GC-MS transfer line, $280^{\circ} \mathrm{C}$; ion source, $230^{\circ} \mathrm{C}$; and quadrupole, $150^{\circ} \mathrm{C}$. The mass-selective detector was operated in scan mode, scanning the mass range from $\mathrm{m} / \mathrm{z} 40$ to 700 . Scan control, data acquisition, and processing were performed by MSD ChemStation and AMDIS software, version 2.69, based on the masses, fragmentation patterns, and retention times, in comparison with Supelco 37 Component FAME Mix and LC-PUFA methyl esters (all Sigma-Aldrich) as reference standards, and NIST 08 database. Absolute amounts of PUFAs were quantified by integration of the peaks using MSD ChemStation and by subsequent calculation in relation to the integral of $n-3$ HPA methyl ester or $n-3$ DPA methyl ester and to CDW.

Reporting summary. Further information on research design is available in the Nature Research Reporting Summary linked to this article.

\section{Data availability}

The sequences of the synthetic PUFA BGCs version C1_V1, C1_V2, C3, and C3_mod 5 have been deposited in the GenBank database (accession numbers MN047805, MN047806, MN047807, and MN047808). The source data underlying Figs. 2, 3, 4, and 5 Supplementary Figs. 12 and 19, and Supplementary Tables 9 and 10 are provided as a Source Data file. All other relevant data are available from the authors upon reasonable request.

Received: 12 March 2019 Accepted: 14 August 2019

Published online: 06 September 2019

\section{References}

1. Madzak, C., Gaillardin, C. \& Beckerich, J. M. Heterologous protein expression and secretion in the non-conventional yeast Yarrowia lipolytica: a review. J. Biotechnol. 109, 63-81 (2004).

2. Nicaud, J. M. et al. Protein expression and secretion in the yeast Yarrowia lipolytica. FEMS Yeast Res. 2, 371-379 (2002).

3. Madzak, C., Treton, B. \& Blanchin-Roland, S. Strong hybrid promoters and integrative expression/secretion vectors for quasi-constitutive expression of heterologous proteins in the yeast Yarrowia lipolytica. J. Mol. Microbiol. Biotechnol. 2, 207-216 (2000).

4. Madzak, C. New tools for heterologous protein production in the yeast Yarrowia lipolytica. Recent Res. Dev. Microbiol. 7, 453-479 (2003).

5. Groenewald, M. et al. Yarrowia lipolytica. Safety assessment of an oleaginous yeast with a great industrial potential. Crit. Rev. Microbiol. 40, 187-206 (2014)

6. Papanikolaou, S., Chevalot, I., Komaitis, M., Marc, I. \& Aggelis, G. Single cell oil production by Yarrowia lipolytica growing on an industrial derivative of animal fat in batch cultures. Appl. Microbiol. Biotechnol. 58, 308-312 (2002).

7. Beopoulos, A. et al. Yarrowia lipolytica as a model for bio-oil production. Prog. Lipid Res. 48, 375-387 (2009).

8. Ratledge, C. Fatty acid biosynthesis in microorganisms being used for single cell oil production. Biochimie 86, 807-815 (2004)

9. Innes, J. K. \& Calder, P. C. The differential effects of eicosapentaenoic acid and docosahexaenoic acid on cardiometabolic risk factors. A Systematic review. Int. J. Mol. Sci. 19, 532 (2018).

10. Xue, Z. X. et al. Production of omega-3 eicosapentaenoic acid by metabolic engineering of Yarrowia lipolytica. Nat. Biotechnol. 31, 734-740 (2013).

11. Damude, H. G. et al. Identification of bifunctional D12/w3 fatty acid desaturases for improving the ratio of w3 to w6 fatty acids in microbes and plants. Proc. Natl Acad. Sci. USA 103, 9446-9451 (2006).

12. Chuang, L. T. et al. Co-expression of heterologous desaturase genes in Yarrowia lipolytica. Nat. Biotechnol. 27, 277-282 (2010).

13. Damude, H. G. et al. Docosahexaenoic acid producing strains of Yarrowia lipolytica. US patent 7,550,286 (2009)

14. Gemperlein, K., Rachid, S., Garcia, R. O., Wenzel, S. C. \& Müller, R. Polyunsaturated fatty acid biosynthesis in myxobacteria. Different PUFA synthases and their product diversity. Chem. Sci. 5, 1733-1741 (2014).

15. Yoshida, K. et al. Bacterial long-chain polyunsaturated fatty acids. Their biosynthetic genes, functions, and practical use. Mar. Drugs 14, https://doi. org/10.3390/md14050094 (2016). 
16. Garcia, R., Stadler, M., Gemperlein, K. \& Müller, R. Aetherobacter fasciculatus gen. nov., sp. nov. and Aetherobacter rufus gen. nov., sp. nov., two novel myxobacteria with promising biotechnological applications. Int. J. Syst. Evol. Microbiol. 66, 928-938 (2016).

17. Garcia, R., Gemperlein, K. \& Müller, R. Minicystis rosea gen. nov., sp. nov., a polyunsaturated fatty acid-rich and steroid-producing soil myxobacterium. Int. J. Syst. Evol. Microbiol. 64, 3733-3742 (2014).

18. Gemperlein, K., Zipf, G., Bernauer, H. S., Müller, R. \& Wenzel, S. C. Metabolic engineering of Pseudomonas putida for production of docosahexaenoic acid based on a myxobacterial PUFA synthase. Metab. Eng. 33, 98-108 (2016).

19. Oßwald, C. et al. Modular construction of a functional artificial epothilone polyketide pathway. ACS Synth. Biol. 3, 759-772 (2014).

20. Bäck, T. \& Schwefel, H. P. An overview of evolutionary algorithms for parameter optimization. Evol. Comput. 1, 1-23 (1993).

21. Goldberg, D. E. Genetic Algorithms in Search, Optimization and Machine Learning. (Addison-Wesley, Boston, 1989).

22. Flagfeldt, D. B., Siewers, V., Huang, L. \& Nielsen, J. Characterization of chromosomal integration sites for heterologous gene expression in Saccharomyces cerevisiae. Yeast 26, 545-551 (2009).

23. Chen, M., Licon, K., Otsuka, R., Pillus, L. \& Ideker, T. Decoupling epigenetic and genetic effects through systematic analysis of gene position. Cell Rep. 3, 128-137 (2013).

24. Janssen, C. I. F. \& Kiliaan, A. J. Long-chain polyunsaturated fatty acids (LCPUFA) from genesis to senescence. The influence of LCPUFA on neural development, aging, and neurodegeneration. Prog. Lipid Res. 53, 1-17 (2014).

25. Strobel, C., Jahreis, G. \& Kuhnt, K. Survey of n-3 and n-6 polyunsaturated fatty acids in fish and fish products. Lipids Health Dis. 11, 144 (2012).

26. Kaur, G., Cameron-Smith, D., Garg, M. \& Sinclair, A. J. Docosapentaenoic acid (22. 5n-3): a review of its biological effects. Prog. Lipid Res. 50, 28-34 (2011).

27. Dujon, B. et al. Genome evolution in yeasts. Nature 430, 35-44 (2004).

28. Grant, S. G., Jessee, J., Bloom, F. R. \& Hanahan, D. Differential plasmid rescue from transgenic mouse DNAs into Escherichia coli methylation-restriction mutants. Proc. Natl Acad. Sci. USA 87, 4645-4649 (1990).

29. Wang, J. et al. An improved recombineering approach by adding RecA to lambda Red recombination. Mol. Biotechnol. 32, 43-53 (2006).

30. Hoffman, C. S. \& Winston, F. A ten-minute DNA preparation from yeast efficiently releases autonomous plasmids for transformation of Escherichia coli. Gene. 57, 267-272 (1987).

31. Bode, H. B. et al. 3-Hydroxy-3-methylglutaryl-coenzyme A (CoA) synthase is involved in the biosynthesis of isovaleryl-CoA in the myxobacterium Myxococcus xanthus during fruiting body formation. J. Bacteriol. 188, 6524-6528 (2006).

32. Bligh, E. G. \& Dyer, W. J. A rapid method of total lipid extraction and purification. Can. J. Biochem. Phys. 37, 911-917 (1959).

33. Lewis, T., Nichols, P. D. \& McMeekin, T. A. Evaluation of extraction methods for recovery of fatty acids from lipid-producing microheterotrophs. $J$. Microbiol. Methods 43, 107-116 (2000).

\section{Acknowledgements}

We thank Nestor Zaburannyi for analyses of Illumina sequence data, Catherine Madzak for plasmid pINA1312, and Isabelle Kocker for support during medium development. This work was generously supported by a grant from the European Union (grant no. C/ 4-EFRE-11/2008), by a grant from the Federal Ministry of Economic Affairs and Energy (grant no. 03SHWB065), and by grants from the Federal Ministry of Education and Research (grant nos. 031B0346A and 031B0346B).

\section{Author contributions}

K.G. performed all cloning experiments in the laboratory and analyzed the GC-MS data from strain evaluation. K.G, G.Z., H.S.B. and S.C.W. designed the artificial DNA sequences. G.Z. and H.S.B. performed the codon adaptation. D.D. and M.K. conducted medium development and fed-batch production of DHA, including all analytics. K.G., C.W., S.C.W. and R.M. designed the study. K.G., D.D., M.K., G.Z., H.S.B., C.W., S.C.W. and R.M. wrote the manuscript.

\section{Additional information}

Supplementary Information accompanies this paper at https://doi.org/10.1038/s41467019-12025-8.

Competing interests: The authors declare no competing interests.

Reprints and permission information is available online at http://npg.nature.com/ reprintsandpermissions/

Peer review information: Nature Communications thanks Martin Grininger and other anonymous reviewer(s) for their contribution to the peer review of this work. Peer reviewer reports are available.

Publisher's note: Springer Nature remains neutral with regard to jurisdictional claims in published maps and institutional affiliations.

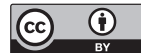

Open Access This article is licensed under a Creative Commons Attribution 4.0 International License, which permits use, sharing, adaptation, distribution and reproduction in any medium or format, as long as you give appropriate credit to the original author(s) and the source, provide a link to the Creative Commons license, and indicate if changes were made. The images or other third party material in this article are included in the article's Creative Commons license, unless indicated otherwise in a credit line to the material. If material is not included in the article's Creative Commons license and your intended use is not permitted by statutory regulation or exceeds the permitted use, you will need to obtain permission directly from the copyright holder. To view a copy of this license, visit http://creativecommons.org/ licenses/by/4.0/.

(C) The Author(s) 2019 